\title{
Dynamical Studies and Numerical Study of the Pandemic Fractional COVID-19 Using the Caputo- Fabrizio Derivative
}

Amr Mahdy ( $\square$ amr_mahdy85@yahoo.com )

Zagazig University Faculty of Science https://orcid.org/0000-0003-2218-5408

\section{Y. A. Amer}

Taif University College of Science

E. S. M. Youssef

Zagazig University Faculty of Science

W. Adel

Zagazig University Faculty of Science

Research article

Keywords: Coronavirus model, Caputo-Fabrizio (CF) fractional derivative; fixed point theorem

Posted Date: April 9th, 2021

DOI: https://doi.org/10.21203/rs.3.rs-387857/v1

License: (9) This work is licensed under a Creative Commons Attribution 4.0 International License.

Read Full License 


\title{
Dynamical studies and numerical study of the pandemic fractional COVID-19 using the Caputo-Fabrizio derivative
}

\author{
A. M. S. Mahdy ${ }^{1,2}$, Y. A. Amer², E. S. M. Youssef ${ }^{2,3}$ and Waleed Adel ${ }^{4}$ \\ ${ }^{1}$ Department of Mathematics, Faculty of Science, Taif University, Saudi Arabia \\ ${ }^{2}$ Department of Mathematics, Faculty of Science, Zagazig University, Zagazig, Egypt \\ ${ }^{3}$ El-Obour Higher Institute of Engineering and Technology, El-Obour, Qalyubia, Egypt \\ ${ }^{4}$ Departement of Mathematics and Engineering Physics, Faculty of Engineering, Mansoura University,
}

Mansoura

amr_mahdy85@yahoo.com ${ }^{1}$,amattaya@tu.edu.sa $a^{2}$,eslamsobhy144@gmail.com ${ }^{3}$, and amrmahdy2282013@gmail.com ${ }^{4}$

\begin{abstract}
In this document, we are in the process of solving and describing the engineering meaning of one of the most dangerous models that affect humans and that spreads at a tremendous speed between people. A Caputo-Fabrizio type partial request numerical model for the fractional Coronavirus model is introduced. The principal properties of the model are investigated. The presence and uniqueness of the answer for the proposed partial Coronavirus model are given through the fixed-point speculation. The mathematical propagations for the model are obtained by using explicit boundary regards. The non-number solicitation subordinate gives continuously versatile and more significant information about the multifaceted idea of the components of the proposed fractional budgetary models of care model than the entire number solicitation models set up beforehand. This new proposed model better may help to better understand the dynamic of this novel virus and may help to better control it.
\end{abstract}

Keywords: Coronavirus model, Caputo-Fabrizio (CF) fractional derivative; fixed point theorem

Mathematics Subject Classification: 41A28, 65D05, 65H10, 65L20, 65P30, 65P40, $65 \mathrm{Z} 05$. 1. Introduction

Outrageous Acute Respiratory Syndrome Coronavirus 2 (SARS-CoV-2) since December 2019 has been seen as one reason for a respiratory ailment. Taking into account that, understanding the changes in a novel and increasing infection erupt is huge anyway testing $[1,2]$. The contamination is assessed to have begun from a fish market in Huanan, Wuhan, and therefore has a zoonotic root [3]. It was set up that $55 \%$ of the spoiled cases inside Wuhan toward the start of tainting were identified with the Seafood Market $[4,5,6]$. The virus is known to be transmitted through small droplets from the nose or mouse when someone sneezes or caught causing the known respiratory infection. These droplets may be considered heavy and may land on other surfaces causing them to be contaminated and other uninfected persons may touch these surfaces and become infected. The major symptoms of this new COVID-19 enlarge a wide range of symptoms including fever, dry caught, and shortness of breathing. These common symptoms occur almost to each infected person and are considered normally mild and gradually increase. One patient out of six may get seriously ill and need to be admitted to the hospital with shortness of breathing. The most affected by the virus if the elder people with underlying health conditions like diabetes, cardiovascular disease, cancer, and chronic respiratory disease.

The continuous, inherited gathering of the human (COVID-19) and that of bat COVID-19 show comparability of $96 \%$. Another zoonotic human Covid that occurred in 2012 is the Middle East Respiratory Syndrome COVID (MERS-CoV). The most generally perceived signs of this disorder fuse the going with: fever, hack, and inconvenience in breathing [7]. Express that a couple of individuals may show non- 
respiratory results like hurling, affliction, and maybe the runs. The consistent COVID-19 erupt has astounded the world in light of the snappy thought of the infection. It has been revealed that by January 23 , an all dwarf of 571 certified cases has been made known in China [8]. A statement was made by the World Health Organization (WHO) on January 30 that the scene of the disorder is a global public health emergency of international concern [4]. As demonstrated by the World Health Organization, the number of people impacted by the novel COVID has beaten 32 million $[9,10]$. Now, in the last month of 2020, the number o confirmed cases infected with the virus has beaten the record of January exceedingly more than 80 million people around the world. The confirmed deaths from the complications of the virus are more than a million and a half. Due to these health-threatening issues, global demand for a cure or a vaccine for this virus is an ongoing process.

Various components have ascribed to the serious provokes looked at by researchers to comprehend the etiology of the COVID-19 pestilence. Right off the bat, the real source of the sickness isn't surely known aside from being connected in a relationship with some wild creatures including a bat. Besides, there is logical proof which demonstrates that commonly the hatching period is inside 2 to 14 days, however, a few people may show no manifestations and could taint others [11]. Furthermore, a few immunizations are being attempted and demonstrated positive towards the spread of the illness, and a few medications are helping in the administration of the COVID-19 pandemic [12]. Treatment that may be utilized in this manner will only focus on enhancing the immune system of the patient to be able to defend against the virus. Other medications may be used to only treat the symptoms until the body begins to defeat the virus.

Fractional order differential equations (FDEs) are commonly utilized to model systems that have a memory that exists in several Physical phenomena, models in the thermoelectricity field, and biological paradigms. FDEs have been utilized to model the realistic biphasic decline manner of elastic systems and infection of diseases but at a slower rate of change. FDEs are more useful than integer-order in modeling sophisticated models that contain physical phenomena. Models with application in engineering, science, physics, and biology are some of the areas that are most used by fractional calculus. Recently, there is some growth in the area of numerical study as well as their applications. For example, a new definition for the fractional calculus was proposed by Catani et. al on [13] without a singular kernel. Also, in [14] a mathematical fractional model of pine disease was investigated with Caputo Fabrizio's definition. In [15] another epidemic model of fractional order in terms of Caputo Fabrizio for simulating the dynamics of hepatitis B is presented. Many other applications of fractional derivative such as cancer treatment model [16], diabetes model [17], and coronavirus model [18-20]. Other relative applications can be found in [21-41] and reference therein.

The subtleties of the rest of the areas of this paper are as per the following: The essential definition and consequences of partial request subsidiary are expressed in Section 2. In Section. 3, we investigate the model definition, model balance, and the essential generation number. Section 4 arrangements with the presence of fragmentary monetary models of mindfulness. Likewise, the uniqueness of a model arrangement is acquired. Numerical reenactments are introduced in Section. 5. At long last, the closing comments are given in Section. 6.

\section{Preliminaries}

Here, we give some essential meanings of the partial analytics that will be utilized in the forward investigation of the model.

Definition 1 Let $f \in H^{(n)}(a, b)$, with $b$ greater than $a, \alpha \in[0,1]$, $a$, then the Caputo-Fabrizio (CF) fractional derivative $[13,14]$ is given as:

$D_{t}^{\alpha}\{f(t)\}=\frac{M(\alpha)}{n-\alpha} \int_{a}^{t} \frac{d^{n}}{d t^{n}} f(\theta) \exp \left[-\frac{\alpha}{n-\alpha}(t-\theta)\right] d \theta, \quad n-1<\alpha \leq n$

Where $M(\alpha)$ is standardization capacity to such an extent that $M(0)=M(1)=1$, However, if $f \notin$ $H^{1}(a, b)$ then we obtained: 
$D_{t}^{\alpha}\{f(t)\}=\frac{\alpha M(\alpha)}{1-\alpha} \int_{a}^{t}(f(t)-f(\theta)) \exp \left[-\frac{\alpha}{1-\alpha}(t-\theta)\right] d \theta$,

Remark 1 if $\sigma=\frac{1-\alpha}{\alpha} \in[0, \infty), \alpha=\frac{1}{1+\sigma} \in[0,1]$, then Eq. (2) gives the following form:

$D_{t}^{\alpha}\{f(t)\}=\frac{N(\sigma)}{\sigma} \int_{a}^{t} f^{\prime}(\theta) \exp \left[-\frac{t-\theta}{\sigma}\right] d \theta, N(0)=N(\infty)=1$

Definition 2 The fractional integral of order $\alpha,(0<\alpha \leq 1)$ of the function $\mathrm{f}(\mathrm{t})$ is defined as:

$I_{t}^{\alpha}\{f(t)\}=\frac{2(1-\alpha)}{(2-\alpha) M(\alpha)} f(t)+\frac{2 \alpha}{(2-\alpha) M(\alpha)} \int_{0}^{t} f(s) d s, \quad t \geq 0$

Remark 2 From Definition 2, we have:

$\frac{2(1-\alpha)}{(2-\alpha) M(\alpha)}+\frac{2 \alpha}{(2-\alpha) M(\alpha)}=1$

Which implies $M(\alpha)=\frac{2}{2-\alpha}, 0<\alpha<1$. Given (5), a new Caputo derivative of order $0<\alpha<1$ is suggested by Nieto and Losada [13], given as follows:

$D_{t}^{\alpha}\{f(t)\}=\frac{1}{1-\alpha} \int_{0}^{t} f^{\prime}(\theta) \exp \left[\frac{\alpha}{1-\alpha}(t-\theta)\right] d \theta$,

The CF subsidiary [13], given in the above definitions, has been as of late utilized in the numerical demonstrating of HBV [15], Maxwell liquid with slip impacts [16], and diabetes model [17].

\section{Model formulation}

This portion presents the Coronavirus model as a changed variation of Khan and Atangana [18] and Chen et al. [19] in which the full scale have vertebrate people $N_{m}$ is partitioned into Susceptible $S_{m}$, Latent warmblooded creature $L_{m}$, Infected very much developed animal $I_{m}$, Recovered warm-blooded animal $R_{m}$. Consequently, the absolute host populace is given by $N_{m}=S_{m}+L_{m}+I_{m}+R_{m}$. The human all out populace is parceled into Susceptible human $S_{h}$, Latent human $L_{h}$, Infected human $I_{h}$ and Recovered human $R_{h}$. The enrollment of pace of warm-blooded animal and human are $A_{m}$ and $A_{h}$ respectively. The characteristic death rate for vertebrates is meant by $\mu_{m}$ and the common mortality of humans is $\mu_{h}$. The successful contact rate that can bring about contamination between Susceptible warm-blooded creatures and Infected mammals $\beta_{1}$ and the suitable contact rate that can accomplish illness between weak human through polluted warmblooded creatures and defiled human is shown by $\beta_{2}, \beta_{3}$ independently. The rate at which recuperated human misfortunes insusceptibility to join the vulnerable class is $\gamma$. The recuperation pace of people and warmblooded creatures are $\tau_{h}$ and $\tau_{m}$ individually. The rate at which human and vertebrate moves into the contaminated class is given by $\theta_{h}$ and $\theta_{m}$ while human illness instigated mortality is $\omega$. It is accepted that there is no deficiency of invulnerability in warm-blooded animals. The accompanying nonlinear differential condition speaks to the collaborations among the different compartments [20]. 


$$
\left\{\begin{array}{l}
\frac{d S_{m}}{d t}=A_{m}-\beta_{1} S_{m} I_{m}-\mu_{m} S_{m} \\
\frac{d L_{m}}{d t}=\beta_{1} S_{m} I_{m}-\left(\mu_{m}+\theta_{m}\right) L_{m} \\
\frac{d I_{m}}{d t}=\theta_{m} L_{m}-\left(\tau_{m}+\mu_{m}\right) I_{m} \\
\frac{d R_{m}}{d t}=\tau_{m} I_{m}-\mu_{m} R_{m} \\
\frac{d S_{h}}{d t}=A_{h}-\beta_{2} S_{h} I_{m}-\beta_{3} S_{h} I_{h}+\gamma R_{h}-\mu_{h} S_{h} \\
\frac{d L_{h}}{d t}=\beta_{2} S_{h} I_{m}+\beta_{3} S_{h} I_{h}-\left(\mu_{h}+\theta_{h}\right) L_{h} \\
\frac{d I_{h}}{d t}=\theta_{h} L_{h}-\left(\tau_{h}+\mu_{h}+\omega\right) I_{h} \\
\frac{d R_{h}}{d t}=\tau_{h} I_{h}-\left(\mu_{h}+\gamma\right) R_{h}
\end{array}\right.
$$

We reformulate the Coronavirus model (7) by displacing the standard entire number solicitation auxiliary by the new CF fragmentary subordinate it might be made as follows[20]:

$$
\left\{\begin{array}{l}
D_{t}^{\alpha} S_{m}=A_{m}-\beta_{1} S_{m} I_{m}-\mu_{m} S_{m} \\
D_{t}^{\alpha} L_{m}=\beta_{1} S_{m} I_{m}-\left(\mu_{m}+\theta_{m}\right) L_{m} \\
D_{t}^{\alpha} I_{m}=\theta_{m} L_{m}-\left(\tau_{m}+\mu_{m}\right) I_{m} \\
D_{t}^{\alpha} R_{m}=\tau_{m} I_{m}-\mu_{m} R_{m} \\
D_{t}^{\alpha} S_{h}=A_{h}-\beta_{2} S_{h} I_{m}-\beta_{3} S_{h} I_{h}+\gamma R_{h}-\mu_{h} S_{h} \\
D_{t}^{\alpha} L_{h}=\beta_{2} S_{h} I_{m}+\beta_{3} S_{h} I_{h}-\left(\mu_{h}+\theta_{h}\right) L_{h} \\
D_{t}^{\alpha} I_{h}=\theta_{h} L_{h}-\left(\tau_{h}+\mu_{h}+\omega\right) I_{h} \\
D_{t}^{\alpha} R_{h}=\tau_{h} I_{h}-\left(\mu_{h}+\gamma\right) R_{h}
\end{array}\right.
$$

With initial conditions:

$$
\begin{aligned}
& S_{m}(0)=S_{m}^{0}, \quad L_{m}(0)=L_{m}^{0}, \quad I_{m}(0)=I_{m}^{0}, \quad R_{m}(0)=R_{m}^{0}, \\
& S_{h}(0)=S_{h}^{0}, \quad L_{h}(0)=L_{h}^{0}, \quad I_{h}(0)=I_{h}^{0}, \quad R_{h}(0)=R_{h}^{0}
\end{aligned}
$$

\section{Existence and uniqueness of fractional Coronavirus model}

This section portrays the presence of model arrangements by utilizing a fixed-point hypothesis. We utilize the fragmentary fundamental administrator in [21] on (8) to acquire:

$$
\left\{\begin{array}{l}
S_{m}(t)-S_{m}(0)={ }_{0}^{C F} I_{t}^{\alpha}\left\{A_{m}-\beta_{1} S_{m} I_{m}-\mu_{m} S_{m}\right\} \\
L_{m}(t)-L_{m}(0)={ }_{0}^{C F} I_{I^{\alpha}}^{\alpha}\left\{\beta_{1} S_{m} I_{m}-\left(\mu_{m}+\theta_{m}\right) L_{m}\right\} \\
I_{m}(t)-I_{m}(0)={ }^{C F}{ }_{0} I_{t}^{\alpha}\left\{\theta_{m} L_{m}-\left(\tau_{m}+\mu_{m}\right) I_{m}\right\} \\
R_{m}(t)-R_{m}(0)={ }^{C}{ }_{0} I_{t}^{\alpha}\left\{\tau_{m} I_{m}-\mu_{m} R_{m}\right\} \\
S_{h}(t)-S_{h}(0)={ }_{0}^{C F} I_{t}^{\alpha}\left\{A_{h}-\beta_{2} S_{h} I_{m}-\beta_{3} S_{h} I_{h}+\gamma R_{h}-\mu_{h} S_{h}\right\} \\
L_{h}(t)-L_{h}(0)={ }_{0}^{C F} I_{t}^{\alpha}\left\{\beta_{2} S_{h} I_{m}+\beta_{3} S_{h} I_{h}-\left(\mu_{h}+\theta_{h}\right) L_{h}\right\} \\
I_{h}(t)-I_{h}(0)={ }_{0}^{C F} I_{t}^{\alpha}\left\{\theta_{h} L_{h}-\left(\tau_{h}+\mu_{h}+\omega\right) I_{h}\right\} \\
R_{h}(t)-R_{h}(0)={ }_{0}^{C F} I_{t}^{\alpha}\left\{\tau_{h} I_{h}-\left(\mu_{h}+\gamma\right) R_{h}\right\}
\end{array}\right.
$$

Applying the thought utilized in [21], we get: 


$$
\begin{aligned}
& \int S_{m}(t)-S_{m}(0)=\frac{2(1-\alpha)}{(2-\alpha) M(\alpha)}\left\{A_{m}-\beta_{1} S_{m} I_{m}-\mu_{m} S_{m}\right\} \\
& +\frac{2 \alpha}{(2-\alpha) M(\alpha)} \int_{0}^{t}\left\{A_{m}-\beta_{1} S_{m} I_{m}-\mu_{m} S_{m}\right\} d s \\
& L_{m}(t)-L_{m}(0)=\frac{2(1-\alpha)}{(2-\alpha) M(\alpha)}\left\{\beta_{1} S_{m} I_{m}-\left(\mu_{m}+\theta_{m}\right) L_{m}\right\} \\
& +\frac{2 \alpha}{(2-\alpha) M(\alpha)} \int_{0}^{t}\left\{\beta_{1} S_{m} I_{m}-\left(\mu_{m}+\theta_{m}\right) L_{m}\right\} d s \\
& I_{m}(t)-I_{m}(0)=\frac{2(1-\alpha)}{(2-\alpha) M(\alpha)}\left\{\theta_{m} L_{m}-\left(\tau_{m}+\mu_{m}\right) I_{m}\right\} \\
& +\frac{2 \alpha}{(2-\alpha) M(\alpha)} \int_{0}^{t}\left\{\theta_{m} L_{m}-\left(\tau_{m}+\mu_{m}\right) I_{m}\right\} d s \\
& R_{m}(t)-R_{m}(0)=\frac{2(1-\alpha)}{(2-\alpha) M(\alpha)}\left\{\tau_{m} I_{m}-\mu_{m} R_{m}\right\} \\
& +\frac{2 \alpha}{(2-\alpha) M(\alpha)} \int_{0}^{t}\left\{\tau_{m} I_{m}-\mu_{m} R_{m}\right\} d s \\
& S_{h}(t)-S_{h}(0)=\frac{2(1-\alpha)}{(2-\alpha) M(\alpha)}\left\{A_{h}-\beta_{2} S_{h} I_{m}-\beta_{3} S_{h} I_{h}+\gamma R_{h}-\mu_{h} S_{h}\right\} \\
& +\frac{2 \alpha}{(2-\alpha) M(\alpha)} \int_{0}^{t}\left\{A_{h}-\beta_{2} S_{h} I_{m}-\beta_{3} S_{h} I_{h}+\gamma R_{h}-\mu_{h} S_{h}\right\} d s \\
& L_{h}(t)-L_{h}(0)=\frac{2(1-\alpha)}{(2-\alpha) M(\alpha)}\left\{\beta_{2} S_{h} I_{m}+\beta_{3} S_{h} I_{h}-\left(\mu_{h}+\theta_{h}\right) L_{h}\right\} \\
& +\frac{2 \alpha}{(2-\alpha) M(\alpha)} \int_{0}^{t}\left\{\beta_{2} S_{h} I_{m}+\beta_{3} S_{h} I_{h}-\left(\mu_{h}+\theta_{h}\right) L_{h}\right\} d s \\
& I_{h}(t)-I_{h}(0)=\frac{2(1-\alpha)}{(2-\alpha) M(\alpha)}\left\{\theta_{h} L_{h}-\left(\tau_{h}+\mu_{h}+\omega\right) I_{h}\right\} \\
& +\frac{2 \alpha}{(2-\alpha) M(\alpha)} \int_{0}^{t}\left\{\theta_{h} L_{h}-\left(\tau_{h}+\mu_{h}+\omega\right) I_{h}\right\} d s \\
& R_{h}(t)-R_{h}(0)=\frac{2(1-\alpha)}{(2-\alpha) M(\alpha)}\left\{\tau_{h} I_{h}-\left(\mu_{h}+\gamma\right) R_{h}\right\} \\
& +\frac{2 \alpha}{(2-\alpha) M(\alpha)} \int_{0}^{t}\left\{\tau_{h} I_{h}-\left(\mu_{h}+\gamma\right) R_{h}\right\} d s
\end{aligned}
$$

For effortlessness, we supplant as follows:

$$
\left\{\begin{array}{l}
F_{1}\left(t, S_{m}\right)=A_{m}-\beta_{1} S_{m} I_{m}-\mu_{m} S_{m} \\
F_{2}\left(t, L_{m}\right)=\beta_{1} S_{m} I_{m}-\left(\mu_{m}+\theta_{m}\right) L_{m} \\
F_{3}\left(t, I_{m}\right)=\theta_{m} L_{m}-\left(\tau_{m}+\mu_{m}\right) I_{m} \\
F_{4}\left(t, R_{m}\right)=\tau_{m} I_{m}-\mu_{m} R_{m} \\
F_{5}\left(t, S_{h}\right)=A_{h}-\beta_{2} S_{h} I_{m}-\beta_{3} S_{h} I_{h}+\gamma R_{h}-\mu_{h} S_{h} \\
F_{6}\left(t, L_{h}\right)=\beta_{2} S_{h} I_{m}+\beta_{3} S_{h} I_{h}-\left(\mu_{h}+\theta_{h}\right) L_{h} \\
F_{7}\left(t, I_{h}\right)=\theta_{h} L_{h}-\left(\tau_{h}+\mu_{h}+\omega\right) I_{h} \\
F_{8}\left(t, R_{h}\right)=\tau_{h} I_{h}-\left(\mu_{h}+\gamma\right) R_{h}
\end{array}\right.
$$


Theorem1 The kernels $F_{1}, F_{2}, F_{3}, F_{4}, F_{5}, F_{6}, F_{7}$ and $F_{8}$ fulfill the Lipschitz condition and withdrawal if the accompanying imbalance holds:

$$
0 \leq \beta_{1} e+\mu_{m}<1
$$

Proof Here, we start from $F_{2}$. Suppose $S_{m}$ and $S_{m 1}$ are two functions, then we assess the following:

$$
\left\|F_{1}\left(t, S_{m}\right)-F_{1}\left(t, S_{m 1}\right)\right\|=\left\|A_{m}-\beta_{1} S_{m} I_{m}-\mu_{m} S_{m}-A_{m}+\beta_{1} S_{m 1} I_{m}+\mu_{m} S_{m 1}\right\|
$$

$\left\|F_{1}\left(t, S_{m}\right)-F_{1}\left(t, S_{m 1}\right)\right\|=\left\|-\beta_{1} I_{m}\left(S_{m}-S_{m 1}\right)-\mu_{m}\left(S-S_{m 1}\right)\right\|$

Using the triangular inequality on Eq. (13), we obtain:

$$
\begin{gathered}
\left\|F_{1}\left(t, S_{m}\right)-F_{1}\left(t, S_{m 1}\right)\right\| \leq \beta_{1}\left\|I_{m}\right\|\left\|S_{m}-S_{m 1}\right\|+\mu_{m}\left\|S_{m}-S_{m 1}\right\| \\
\left\|F_{1}\left(t, S_{m}\right)-F_{1}\left(t, S_{m 1}\right)\right\| \leq\left[\beta_{1}\left\|I_{m}\right\|+\mu_{m}\right]\left\|S_{m}-S_{m 1}\right\| \\
\left\|F_{1}\left(t, S_{m}\right)-F_{1}\left(t, S_{m 1}\right)\right\| \leq\left[\beta_{1} e+\mu_{m}\right]\left\|S_{m}-S_{m 1}\right\|
\end{gathered}
$$

By taking that $\beta_{1} e+\mu_{m}=\mu_{1}$, where $\left\|I_{m}\right\| \leq e$ is a bounded function, we get:

$\left\|F_{1}\left(t, S_{m}\right)-F_{1}\left(t, S_{m 1}\right)\right\| \leq \mu_{1}\left\|S_{m}-S_{m 1}\right\|$

Hence, the Lipschitz condition is fulfilled for $F_{2}$ and if also $0 \leq b_{1}+b_{2}+b_{3}+\gamma_{2}<1$ then it is also a contraction. For the rest of the cases, likewise, the Lipschitz conditions are given as follows:

$$
\left\{\begin{aligned}
\left\|F_{2}\left(t, L_{m}\right)-F_{2}\left(t, L_{m 1}\right)\right\| & \leq \mu_{2}\left\|L_{m}-L_{m 1}\right\| \\
\left\|F_{3}\left(t, I_{m}\right)-F_{3}\left(t, I_{m 1}\right)\right\| & \leq \mu_{3}\left\|I_{m}-I_{m 1}\right\| \\
\left\|F_{4}\left(t, R_{m}\right)-F_{4}\left(t, R_{m 1}\right)\right\| & \leq \mu_{4}\left\|R_{m}-R_{m 1}\right\| \\
\left\|F_{5}\left(t, S_{h}\right)-F_{5}\left(t, S_{h 1}\right)\right\| & \leq \mu_{5}\left\|S_{h}-S_{h 1}\right\| \\
\left\|F_{6}\left(t, L_{h}\right)-F_{6}\left(t, L_{h 1}\right)\right\| & \leq \mu_{6}\left\|L_{h}-L_{h 1}\right\| \\
\left\|F_{7}\left(t, I_{h}\right)-F_{7}\left(t, I_{h 1}\right)\right\| & \leq \mu_{7}\left\|I_{h}-I_{h 1}\right\| \\
\left\|F_{8}\left(t, R_{h}\right)-F_{8}\left(t, R_{h 1}\right)\right\| & \leq \mu_{8}\left\|R_{h}-R_{h 1}\right\|
\end{aligned}\right.
$$

Utilizing documentation for parts, Eq. (10) becomes: 


$$
\left\{\begin{array}{l}
S_{m}(t)=S_{m}(0)+\frac{2(1-\alpha)}{(2-\alpha) M(\alpha)} F_{1}\left(t, S_{m}\right)+\frac{2 \alpha}{(2-\alpha) M(\alpha)} \int_{0}^{t} F_{1}\left(s, S_{m}\right) d s \\
L_{m}(t)=L_{m}(0)+\frac{2(1-\alpha)}{(2-\alpha) M(\alpha)} F_{3}\left(t, I_{m}\right)+\frac{2 \alpha}{(2-\alpha) M(\alpha)} \int_{0}^{t} F_{3}\left(s, I_{m}\right) d s \\
I_{m}(t)=I_{m}(0)+\frac{2(1-\alpha)}{(2-\alpha) M(\alpha)} F_{3}\left(t, I_{m}\right)+\frac{2 \alpha}{(2-\alpha) M(\alpha)} \int_{0}^{t} F_{3}\left(s, I_{m}\right) d s \\
R_{m}(t)=R_{m}(0)+\frac{2(1-\alpha)}{(2-\alpha) M(\alpha)} F_{4}\left(t, R_{m}\right)+\frac{2 \alpha}{(2-\alpha) M(\alpha)} \int_{0}^{t} F_{4}\left(s, R_{m}\right) d s \\
S_{h}(t)=S_{h}(0)+\frac{2(1-\alpha)}{(2-\alpha) M(\alpha)} F_{5}\left(t, S_{h}\right)+\frac{2 \alpha}{(2-\alpha) M(\alpha)} \int_{0}^{t} F_{5}\left(s, S_{h}\right) d s \\
L_{h}(t)=L_{h}(0)+\frac{2(1-\alpha)}{(2-\alpha) M(\alpha)} F_{6}\left(t, L_{h}\right)+\frac{2 \alpha}{(2-\alpha) M(\alpha)} \int_{0}^{t} F_{6}\left(s, L_{h}\right) d s \\
I_{h}(t)=I_{h}(0)+\frac{2(1-\alpha)}{(2-\alpha) M(\alpha)} F_{7}\left(t, I_{h}\right)+\frac{2 \alpha}{(2-\alpha) M(\alpha)} \int_{0}^{t} F_{7}\left(s, I_{h}\right) d s \\
R_{h}(t)=R_{h}(0)+\frac{2(1-\alpha)}{(2-\alpha) M(\alpha)} F_{8}\left(t, R_{h}\right)+\frac{2 \alpha}{(2-\alpha) M(\alpha)} \int_{0}^{t} F_{3}\left(s, R_{m}\right) d s
\end{array}\right.
$$

The accompanying recursive recipe is introduced:

$$
\left\{\begin{array}{l}
S_{m n}(t)=\frac{2(1-\alpha)}{(2-\alpha) M(\alpha)} F_{1}\left(t, S_{m(n-1)}\right)+\frac{2 \alpha}{(2-\alpha) M(\alpha)} \int_{0}^{t} F_{1}\left(s, S_{m(n-1)}\right) d s \\
L_{m n}(t)=\frac{2(1-\alpha)}{(2-\alpha) M(\alpha)} F_{3}\left(t, I_{m(n-1)}\right)+\frac{2 \alpha}{(2-\alpha) M(\alpha)} \int_{0}^{t} F_{3}\left(s, I_{m(n-1)}\right) d s \\
I_{m n}(t)=\frac{2(1-\alpha)}{(2-\alpha) M(\alpha)} F_{3}\left(t, I_{m(n-1)}\right)+\frac{2 \alpha}{(2-\alpha) M(\alpha)} \int_{0}^{t} F_{3}\left(s, I_{m(n-1)}\right) d s \\
R_{m n}(t)=\frac{2(1-\alpha)}{(2-\alpha) M(\alpha)} F_{4}\left(t, R_{m(n-1)}\right)+\frac{2 \alpha}{(2-\alpha) M(\alpha)} \int_{0}^{t} F_{4}\left(s, R_{m(n-1)}\right) d s \\
S_{h n}(t)=\frac{2(1-\alpha)}{(2-\alpha) M(\alpha)} F_{5}\left(t, S_{h(n-1)}\right)+\frac{2 \alpha}{(2-\alpha) M(\alpha)} \int_{0}^{t} F_{5}\left(s, S_{h(n-1)}\right) d s \\
L_{h n}(t)=\frac{2(1-\alpha)}{(2-\alpha) M(\alpha)} F_{6}\left(t, L_{h(n-1)}\right)+\frac{2 \alpha}{(2-\alpha) M(\alpha)} \int_{0}^{t} F_{6}\left(s, L_{h(n-1)}\right) d s \\
I_{h n}(t)=\frac{2(1-\alpha)}{(2-\alpha) M(\alpha)} F_{7}\left(t, I_{h(n-1)}\right)+\frac{2 \alpha}{(2-\alpha) M(\alpha)} \int_{0}^{t} F_{7}\left(s, I_{h(n-1)}\right) d s \\
R_{h n}(t)=\frac{2(1-\alpha)}{(2-\alpha) M(\alpha)} F_{8}\left(t, R_{h(n-1)}\right)+\frac{2 \alpha}{(2-\alpha) M(\alpha)} \int_{0}^{t} F_{8}\left(s, R_{h(n-1)}\right) d s
\end{array}\right.
$$


with the initial conditions:

$$
\left\{\begin{array}{ccl}
S_{m}(0)=S_{m}^{0}(t), & L_{m}(0)=L_{m}^{0}(t), \quad I_{m}(0)=I_{m}^{0}(t), \quad R_{m}(0)=R_{m}^{0}(t) \\
S_{h}(0)=S_{h}^{0}(t), & L_{h}(0)=L_{h}^{0}(t), \quad I_{h}(0)=I_{h}^{0}(t), \quad R_{h}(0)=R_{h}^{0}(t)
\end{array}\right.
$$

The contrast between the progressive terms is determined as follows:

$$
\begin{aligned}
& \int \omega_{1 n}(t)=S_{m n}(t)-S_{m(n-1)}(t)=\frac{2(1-\alpha)}{(2-\alpha) M(\alpha)}\left[F_{1}\left(t, S_{m(n-1)}\right)-F_{1}\left(t, S_{m(n-2)}\right)\right] \\
& +\frac{2 \alpha}{(2-\alpha) M(\alpha)} \int_{0}^{t}\left[F_{1}\left(s, S_{m(n-1)}\right)-F_{1}\left(s, S_{m(n-2)}\right)\right] d s \\
& \omega_{2 n}(t)=L_{m n}(t)-L_{m(n-1)}(t)=\frac{2(1-\alpha)}{(2-\alpha) M(\alpha)}\left[F_{2}\left(t, L_{m(n-1)}\right)-F_{2}\left(t, L_{m(n-2)}\right)\right] \\
& +\frac{2 \alpha}{(2-\alpha) M(\alpha)} \int_{0}^{t}\left[F_{2}\left(s, L_{m(n-1)}\right)-F_{2}\left(s, L_{m(n-2)}\right)\right] d s \\
& \omega_{3 n}(t)=I_{m n}(t)-I_{m(n-1)}(t)=\frac{2(1-\alpha)}{(2-\alpha) M(\alpha)}\left[F_{3}\left(t, I_{m(n-1)}\right)-F_{3}\left(t, I_{m(n-2)}\right)\right] \\
& +\frac{2 \alpha}{(2-\alpha) M(\alpha)} \int_{0}^{t}\left[F_{3}\left(s, I_{m(n-1)}\right)-F_{3}\left(s, I_{m(n-2)}\right)\right] d s \\
& \omega_{4 n}(t)=R_{m n}(t)-R_{m(n-1)}(t)=\frac{2(1-\alpha)}{(2-\alpha) M(\alpha)}\left[F_{4}\left(t, R_{m(n-1)}\right)-F_{4}\left(t, R_{m(n-2)}\right)\right] \\
& +\frac{2 \alpha}{(2-\alpha) M(\alpha)} \int_{0}^{t}\left[F_{4}\left(s, R_{m(n-1)}\right)-F_{4}\left(s, R_{m(n-2)}\right)\right] d s \\
& \omega_{5 n}(t)=S_{h n}(t)-S_{h(n-1)}(t)=\frac{2(1-\alpha)}{(2-\alpha) M(\alpha)}\left[F_{5}\left(t, S_{h(n-1)}\right)-F_{5}\left(t, S_{h(n-2)}\right)\right] \\
& +\frac{2 \alpha}{(2-\alpha) M(\alpha)} \int_{0}^{t}\left[F_{5}\left(s, S_{h(n-1)}\right)-F_{5}\left(s, S_{h(n-2)}\right)\right] d s \\
& \omega_{6 n}(t)=L_{h n}(t)-L_{h(n-1)}(t)=\frac{2(1-\alpha)}{(2-\alpha) M(\alpha)}\left[F_{6}\left(t, L_{h(n-1)}\right)-F_{6}\left(t, L_{h(n-2)}\right)\right] \\
& +\frac{2 \alpha}{(2-\alpha) M(\alpha)} \int_{0}^{t}\left[F_{6}\left(s, L_{h(n-1)}\right)-F_{6}\left(s, L_{h(n-2)}\right)\right] d s \\
& \omega_{7 n}(t)=I_{h n}(t)-I_{h(n-1)}(t)=\frac{2(1-\alpha)}{(2-\alpha) M(\alpha)}\left[F_{7}\left(t, I_{h(n-1)}\right)-F_{7}\left(t, I_{h(n-2)}\right)\right] \\
& +\frac{2 \alpha}{(2-\alpha) M(\alpha)} \int_{0}^{t}\left[F_{7}\left(s, I_{h(n-1)}\right)-F_{7}\left(s, I_{h(n-2)}\right)\right] d s \\
& \omega_{8 n}(t)=R_{h n}(t)-R_{h(n-1)}(t)=\frac{2(1-\alpha)}{(2-\alpha) M(\alpha)}\left[F_{8}\left(t, R_{h(n-1)}\right)-F_{8}\left(t, R_{h(n-2)}\right)\right] \\
& +\frac{2 \alpha}{(2-\alpha) M(\alpha)} \int_{0}^{t}\left[F_{8}\left(s, R_{h(n-1)}\right)-F_{8}\left(s, R_{h(n-2)}\right)\right] d s
\end{aligned}
$$

Notice that 


$$
\left\{\begin{array}{l}
S_{m n}(t)=\sum_{i=1}^{n} \omega_{1 i}(t), L_{m n}(t)=\sum_{i=1}^{n} \omega_{2 i}(t), I_{m n}(t)=\sum_{i=1}^{n} \omega_{3 i}(t), R_{m n}(t)=\sum_{i=1}^{n} \omega_{4 i}(t) \\
S_{h n}(t)=\sum_{i=1}^{n} \omega_{5 i}(t), L_{h n}(t)=\sum_{i=1}^{n} \omega_{6 i}(t), I_{h n}(t)=\sum_{i=1}^{n} \omega_{7 i}(t), R_{h n}(t)=\sum_{i=1}^{n} \omega_{8 i}(t)
\end{array}\right.
$$

On proceeding with a similar procedure, we survey

$$
\begin{aligned}
& \left\|\omega_{1 n}(t)\right\|=\left\|S_{m n}(t)-S_{m(n-1)}(t)\right\|= \\
& \left\|\frac{2(1-\alpha)}{(2-\alpha) M(\alpha)}\left[F_{1}\left(t, S_{m(n-1)}\right)-F_{1}\left(t, S_{m(n-2)}\right)\right]+\frac{2 \alpha}{(2-\alpha) M(\alpha)} \int_{0}^{t}\left[F_{1}\left(s, S_{m(n-1)}\right)-F_{1}\left(s, S_{m(n-2)}\right)\right] d s\right\|
\end{aligned}
$$

Using the triangular inequality, Eq. (21) is simplified to

$$
\begin{aligned}
& \left\|S_{m n}(t)-S_{m(n-1)}(t)\right\| \leq \frac{2(1-\alpha)}{(2-\alpha) M(\alpha)}\left\|F_{1}\left(t, S_{m(n-1)}\right)-F_{1}\left(t, S_{m(n-2)}\right)\right\| \\
& \quad+\frac{2 \alpha}{(2-\alpha) M(\alpha)}\left\|\int_{0}^{t}\left[F_{1}\left(s, S_{m(n-1)}\right)-F_{1}\left(s, S_{m(n-2)}\right)\right] d s\right\|
\end{aligned}
$$

As the kernel fulfills the Lipschitz condition, then we have

$$
\begin{aligned}
\left\|S_{m n}(t)-S_{m(n-1)}(t)\right\| \leq \frac{2(1-\alpha)}{(2-\alpha) M(\alpha)} \mu_{1}\left\|S_{m(n-1)}-S_{m(n-2)}\right\| \\
+\frac{2 \alpha}{(2-\alpha) M(\alpha)} \mu_{1} \int_{0}^{t}\left\|S_{m(n-1)}-S_{m(n-2)}\right\| d s
\end{aligned}
$$

Then we have

$$
\left\|\omega_{1 n}(t)\right\| \leq \frac{2(1-\alpha)}{(2-\alpha) M(\alpha)} \mu_{1}\|\| \omega_{1(n-1)}(t)\|\|+\frac{2 \alpha}{(2-\alpha) M(\alpha)} \mu_{1} \int_{0}^{t}\left\|\omega_{1(n-1)}(s)\right\| d s
$$

Likewise, we get the accompanying outcomes: 


$$
\begin{aligned}
& \left\|\omega_{2 n}(t)\right\| \leq \frac{2(1-\alpha)}{(2-\alpha) M(\alpha)} \mu_{2}\|\| \omega_{2(n-1)}(t)\|\|+\frac{2 \alpha}{(2-\alpha) M(\alpha)} \mu_{2} \int_{0}^{t}\left\|\omega_{2(n-1)}(s)\right\| d s \\
& \left\|\omega_{3 n}(t)\right\| \leq \frac{2(1-\alpha)}{(2-\alpha) M(\alpha)} \mu_{3}\|\| \omega_{3(n-1)}(t)\|\|+\frac{2 \alpha}{(2-\alpha) M(\alpha)} \mu_{3} \int_{0}^{t}\left\|\omega_{3(n-1)}(s)\right\| d s \\
& \left\|\omega_{4 n}(t)\right\| \leq \frac{2(1-\alpha)}{(2-\alpha) M(\alpha)} \mu_{4}\|\| \omega_{4(n-1)}(t)\|\|+\frac{2 \alpha}{(2-\alpha) M(\alpha)} \mu_{4} \int_{0}^{t}\left\|\omega_{4(n-1)}(s)\right\| d s \\
& \left\|\omega_{5 n}(t)\right\| \leq \frac{2(1-\alpha)}{(2-\alpha) M(\alpha)} \mu_{5}\|\| \omega_{5(n-1)}(t)\|\|+\frac{2 \alpha}{(2-\alpha) M(\alpha)} \mu_{5} \int_{0}^{t}\left\|\omega_{5(n-1)}(s)\right\| d s \\
& \left\|\omega_{6 n}(t)\right\| \leq \frac{2(1-\alpha)}{(2-\alpha) M(\alpha)} \mu_{6}\|\| \omega_{6(n-1)}(t)\|\|+\frac{2 \alpha}{(2-\alpha) M(\alpha)} \mu_{6} \int_{0}^{t}\left\|\omega_{6(n-1)}(s)\right\| d s \\
& \left\|\omega_{7 n}(t)\right\| \leq \frac{2(1-\alpha)}{(2-\alpha) M(\alpha)} \mu_{7}\|\| \omega_{7(n-1)}(t)\|\|+\frac{2 \alpha}{(2-\alpha) M(\alpha)} \mu_{7} \int_{0}^{t}\left\|\omega_{7(n-1)}(s)\right\| d s \\
& \left\|\omega_{8 n}(t)\right\| \leq \frac{2(1-\alpha)}{(2-\alpha) M(\alpha)} \mu_{8}\|\| \omega_{8(n-1)}(t)\|\|+\frac{2 \alpha}{(2-\alpha) M(\alpha)} \mu_{8} \int_{0}^{t}\left\|\omega_{8(n-1)}(s)\right\| d s
\end{aligned}
$$

Now we state the theorem below.

Theorem 2 The fractional breast cancer model (8) has precise coupled arrangements if the conditions underneath hold. That is, we can discover $t_{0}$ with the end goal that

$$
\frac{2(1-\alpha)}{(2-\alpha) M(\alpha)} \mu_{1}+\frac{2 \alpha}{(2-\alpha) M(\alpha)} \mu_{1} t_{0}<1
$$

Proof Since all the functions $S_{m}(t), L_{m}(t), I_{m}(t), R_{m}(t), S_{h}(t), L_{h}(t), I_{h}(t)$ and $R_{h}(t)$ are limited, we have demonstrated that the pieces satisfy the Lipschitz condition, hence on utilizing Eqs. (24) and Eqs. (25) and by utilizing the recursive technique, we get the succeeding connection as follow

$$
\begin{aligned}
&\left\|\omega_{1 n}(t)\right\| \leq\left\|S_{m n}(0)\right\|\left[\frac{2(1-\alpha)}{(2-\alpha) M(\alpha)} \mu_{1}+\frac{2(1-\alpha)}{(2-\alpha) M(\alpha)} \mu_{1} t\right]^{n} \\
&\left\|\omega_{2 n}(t)\right\| \leq\left\|L_{m n}(0)\right\|\left[\frac{2(1-\alpha)}{(2-\alpha) M(\alpha)} \mu_{2}+\frac{2(1-\alpha)}{(2-\alpha) M(\alpha)} \mu_{2} t\right]^{n} \\
&\left\|\omega_{3 n}(t)\right\| \leq\left\|I_{m n}(0)\right\|\left[\frac{2(1-\alpha)}{(2-\alpha) M(\alpha)} \mu_{3}+\frac{2(1-\alpha)}{(2-\alpha) M(\alpha)} \mu_{3} t\right]^{n} \\
&\left\|\omega_{4 n}(t)\right\| \leq\left\|R_{m n}(0)\right\|\left[\frac{2(1-\alpha)}{(2-\alpha) M(\alpha)} \mu_{4}+\frac{2(1-\alpha)}{(2-\alpha) M(\alpha)} \mu_{4} t\right]^{n} \\
&\left\|\omega_{5 n}(t)\right\| \leq\left\|S_{h n}(0)\right\|\left[\frac{2(1-\alpha)}{(2-\alpha) M(\alpha)} \mu_{5}+\frac{2(1-\alpha)}{(2-\alpha) M(\alpha)} \mu_{5} t\right]^{n} \\
&\left\|\omega_{6 n}(t)\right\| \leq\left\|L_{h n}(0)\right\|\left[\frac{2(1-\alpha)}{(2-\alpha) M(\alpha)} \mu_{6}+\frac{2(1-\alpha)}{(2-\alpha) M(\alpha)} \mu_{6} t\right]^{n} \\
&\left\|\omega_{7 n}(t)\right\| \leq\left\|I_{h n}(0)\right\|\left[\frac{2(1-\alpha)}{(2-\alpha) M(\alpha)} \mu_{7}+\frac{2(1-\alpha)}{(2-\alpha) M(\alpha)} \mu_{7} t\right]^{n} \\
&\left\|\omega_{8 n}(t)\right\| \leq\left\|R_{h n}(0)\right\|\left[\frac{2(1-\alpha)}{(2-\alpha) M(\alpha)} \mu_{8}+\frac{2(1-\alpha)}{(2-\alpha) M(\alpha)} \mu_{8} t\right]^{n}
\end{aligned}
$$


Hence, the existence and continuity of the said solutions are proved. Furthermore, to ensure that the above function is a solution of Eq. (9), we proceed as follows:

$$
\begin{aligned}
S_{m}(t)-S_{m}(0) & =S_{m n}(t)-A_{n}(t) \\
L_{m}(t)-L_{m}(0) & =L_{m n}(t)-B_{n}(t) \\
I_{m}(t)-I_{m}(0) & =I_{m n}(t)-C_{n}(t) \\
R_{m}(t)-R_{m}(0) & =R_{m n}(t)-D_{n}(t) \\
S_{h}(t)-S_{h}(0) & =S_{h n}(t)-E_{n}(t) \\
L_{h}(t)-L_{h}(0) & =L_{h n}(t)-F_{n}(t) \\
I_{h}(t)-I_{h}(0) & =I_{h n}(t)-G_{n}(t) \\
R_{h}(t)-R_{h}(0) & =R_{h n}(t)-H_{n}(t)
\end{aligned}
$$

Therefore, we have:

$$
\begin{gathered}
\left\|A_{n}(t)\right\|=\left\|\frac{2(1-\alpha)}{(2-\alpha) M(\alpha)}\left[F_{1}\left(s, S_{m n}\right)-F_{1}\left(s, S_{m(n-1)}\right)\right]+\frac{2 \alpha}{(2-\alpha) M(\alpha)} \int_{0}^{t}\left[F_{1}\left(s, S_{m n}\right)-F_{1}\left(s, S_{m(n-1)}\right)\right] d s\right\| \\
\left\|A_{n}(t)\right\| \leq \frac{2(1-\alpha)}{(2-\alpha) M(\alpha)}\left\|F_{1}\left(t, S_{m n}\right)-F_{1}\left(t, S_{m(n-1)}\right)\right\|+\frac{2 \alpha}{(2-\alpha) M(\alpha)} \int_{0}^{t}\left\|F_{1}\left(t, S_{m n}\right)-F_{1}\left(t, S_{m(n-1)}\right)\right\| d s \\
\left\|A_{n}(t)\right\| \leq \frac{2(1-\alpha)}{(2-\alpha) M(\alpha)} \mu_{1}\left\|S_{m n}-S_{m(n-1)}\right\|+\frac{2 \alpha}{(2-\alpha) M(\alpha)} \mu_{1}\left\|, S_{m n}-S_{m(n-1)}\right\| t
\end{gathered}
$$

Recursively using the process gives:

$$
\left\|A_{n}(t)\right\| \leq\left(\frac{2(1-\alpha)}{(2-\alpha) M(\alpha)}+\frac{2 \alpha}{(2-\alpha) M(\alpha)} t\right)^{n+1} \mu_{1}^{n+1} a
$$

Then at $t_{0}$ we have:

$$
\left\|A_{n}(t)\right\| \leq\left(\frac{2(1-\alpha)}{(2-\alpha) M(\alpha)}+\frac{2 \alpha}{(2-\alpha) M(\alpha)} t_{0}\right)^{n+1} \mu_{1}^{n+1} a
$$

By applying the limit on Eq. (30) as $n$ tends to infinity, we get:

$$
\left\|A_{n}(t)\right\| \rightarrow 0
$$

Similarly, we get

$$
\left\|B_{n}(t)\right\| \rightarrow 0,\left\|C_{n}(t)\right\| \rightarrow 0,\left\|D_{n}(t)\right\| \rightarrow 0, \quad\left\|E_{n}(t)\right\| \rightarrow 0,\left\|F_{n}(t)\right\| \rightarrow 0,\left\|G_{n}(t)\right\| \rightarrow 0,\left\|H_{n}(t)\right\| \rightarrow 0
$$

For the uniqueness system (8) solution, we take on the contrary that there exists another solution of (8) given by $S_{m 1}(t), L_{m 1}(t), I_{m 1}(t), R_{m 1}(t), S_{h 1}(t), L_{h 1}(t), I_{h 1}(t)$ and $R_{h 1}(t)$ Then

$$
S_{m}(t)-S_{m 1}(t)=\frac{2(1-\alpha)}{(2-\alpha) M(\alpha)}\left[F_{1}\left(t, S_{m}\right)-F_{1}\left(t, S_{m 1}\right)\right]+\frac{2 \alpha}{(2-\alpha) M(\alpha)} \int_{0}^{t}\left[F_{1}\left(s, S_{m}\right)-F_{1}\left(s, S_{m 1}\right)\right] d s
$$

Taking norm on Eq. (31), we get 
$\left\|S_{m}(t)-S_{m 1}(t)\right\| \leq \frac{2(1-\alpha)}{(2-\alpha) M(\alpha)}\left\|F_{1}\left(t, S_{m}\right)-F_{1}\left(t, S_{m 1}\right)\right\|+\frac{2 \alpha}{(2-\alpha) M(\alpha)} \int_{0}^{t}\left\|F_{1}\left(s, S_{m}\right)-F_{1}\left(s, S_{m 1}\right)\right\| d s$

By applying the Lipschitz condition of the kernel, we have:

$\left\|S_{m}(t)-S_{m 1}(t)\right\| \leq \frac{2(1-\alpha)}{(2-\alpha) M(\alpha)} \mu_{1}\left\|S_{m}-S_{m 1}\right\|+\frac{2 \alpha}{(2-\alpha) M(\alpha)} \int_{0}^{t} \mu_{1} t\left\|S_{m}-S_{m 1}\right\| d s$

It gives that:

$$
\left\|S_{m}(t)-S_{m 1}(t)\right\|\left(1-\frac{2(1-\alpha)}{(2-\alpha) M(\alpha)} \mu_{1}-\frac{2 \alpha}{(2-\alpha) M(\alpha)} \mu_{1} t\right) \leq 0
$$

Theorem 3 The model (8) solution will be unique if

$$
\left(1-\frac{2(1-\alpha)}{(2-\alpha) M(\alpha)} \mu_{1}-\frac{2 \alpha}{(2-\alpha) M(\alpha)} \mu_{1} t\right)>0
$$

Proof If condition (35) holds, then (34) implies that

$$
\left\|S_{m}(t)-S_{m 1}(t)\right\|=0
$$

Hence, we get

$$
S_{m}(t)=S_{m 1}(t)
$$

On employing the same procedure, we get:

\section{Simulation Results}

$$
\begin{aligned}
& L_{m}(t)=L_{m 1}(t), \quad I_{m}(t)=I_{m 1}(t), \quad R_{m}(t)=R_{m 1}(t) \\
& S_{h}(t)=S_{h 1}, \quad L_{h}(t)=L_{h 1}, \quad I_{h}(t)=I_{h 1}, \quad R_{h}(t)=R_{h 1}
\end{aligned}
$$

In this section, we get mathematical outcomes that demonstrate the presence of the method plot. We are actualized the improved Adams-Bashforth-Moulton given for mathematical simulation see [28, 37-39]. Here, we resolve the fractional-order corvid-19 model numerically helping the predictor-corrector PECE method of Adams-Bashforth-Moulton kind qualified in specified in [28, 37-39]. Also, we are present numerical simulations to explain the key aspects of the growth of the analysis of COVID-19. Figures 1-4 explains a model story for the dynamical manner of COVID-19. The extent of the virus develops exponentially until much of the population is infective or recovered, at which point the danger of infections begins to decline. Also, we are showing the relationship between the variables. Here, from the outcomes and the figures mentioned, we ability nation that decreasing the fractional derivative order alpha decreases the number of each people point (save Susceptible people fraction as expected), and press the curves also delays reaching the utmost in all people phase. 


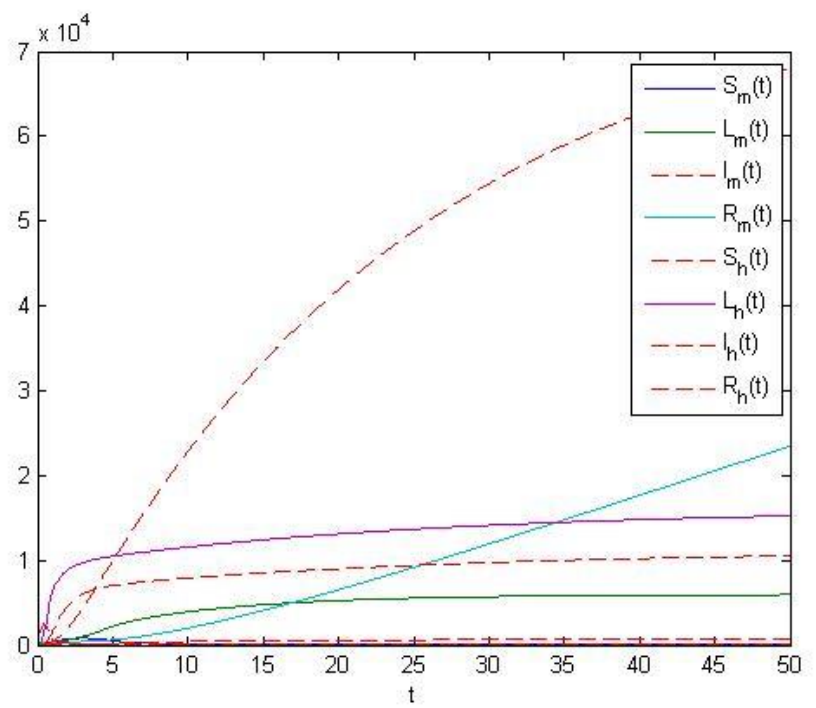

Fig. 1 Different value of the parameters for $\alpha=0.85$ and for $0<t<50$.

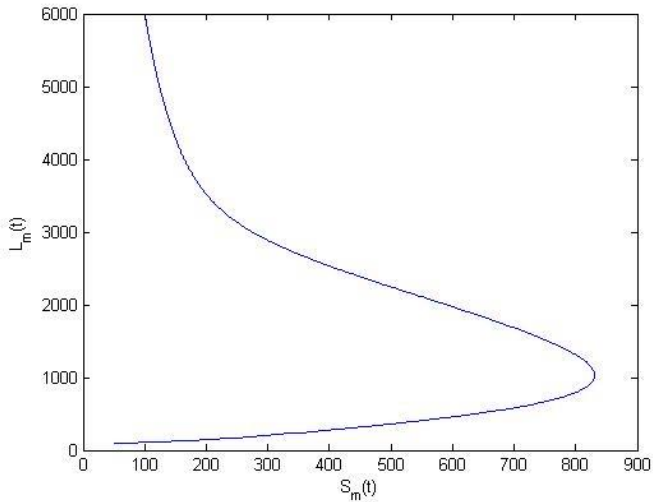

(a)

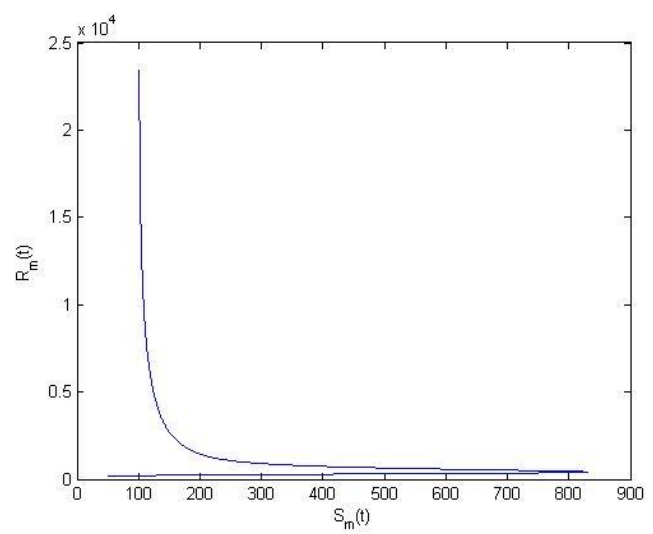

(c)

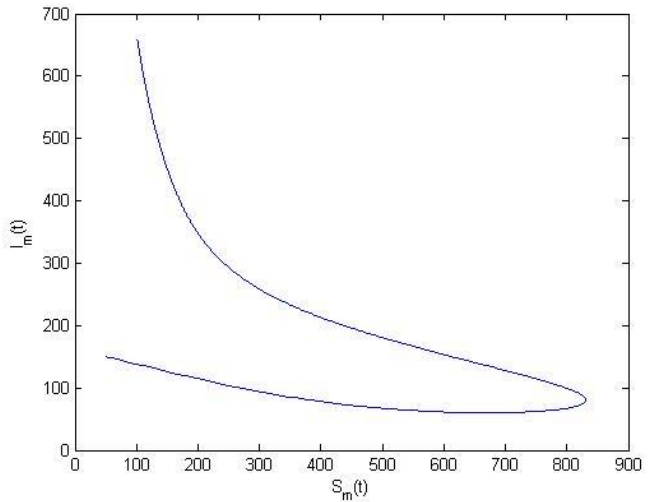

(b)

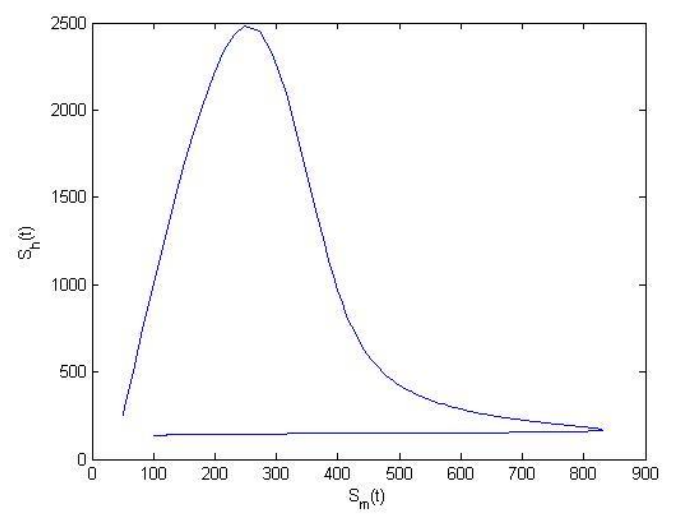

(d) 


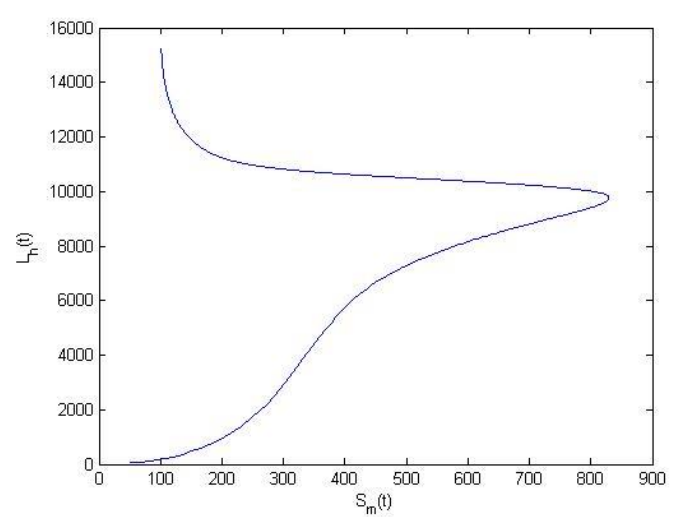

(e)

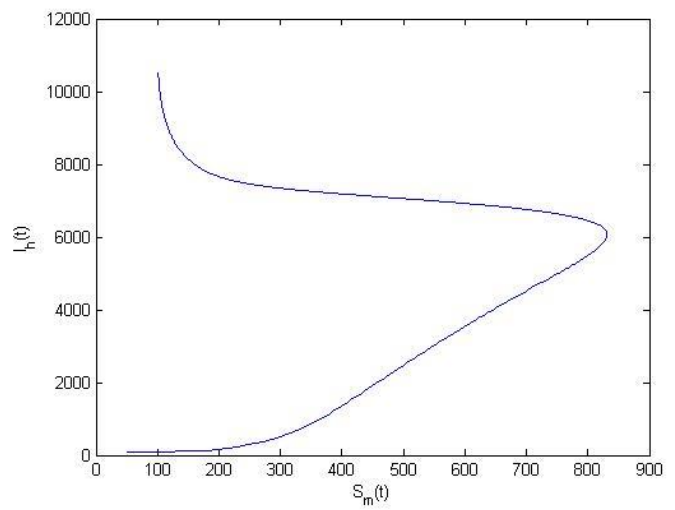

(f)

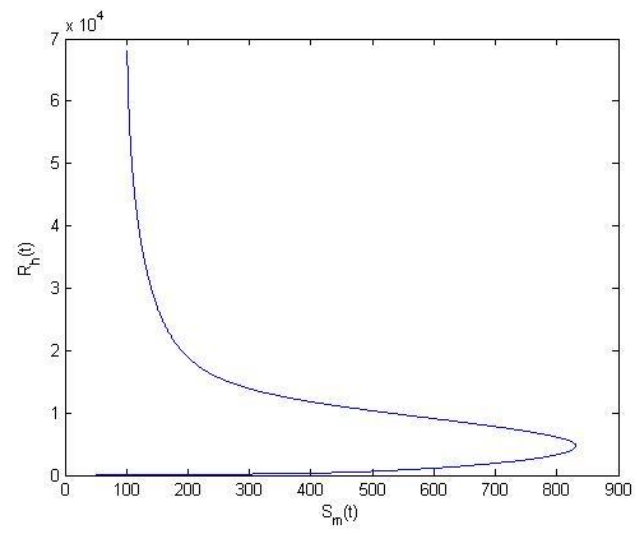

(g)

Fig. 2 Graphical behavior of (a) $L_{m}(t)$, (b) $I_{m}(t)$, (c) $R_{m}(t)$, (d) $S_{h}(t)$, (e) $L_{h}(t)$, (f) $I_{h}(t)$ and (g) $R_{h}(t)$ with $S_{m}(t)$ for $\alpha=0.85$ and for $0<t<900$.

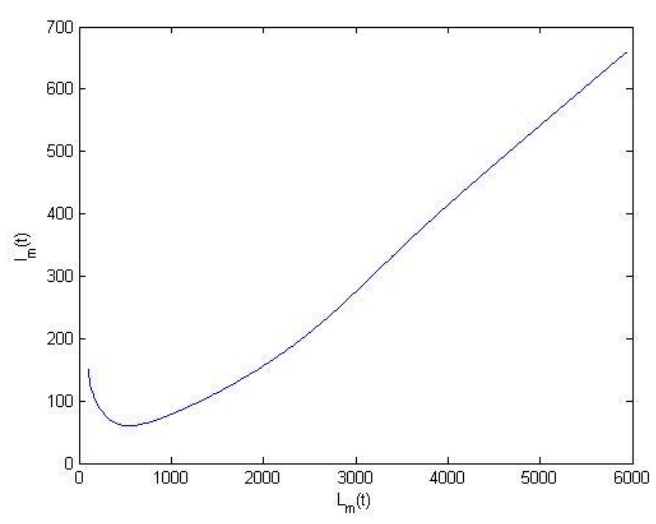

(h)

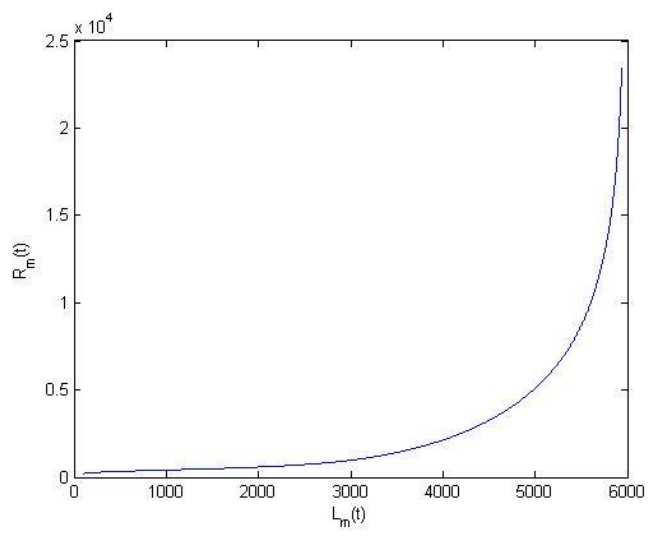

(i) 


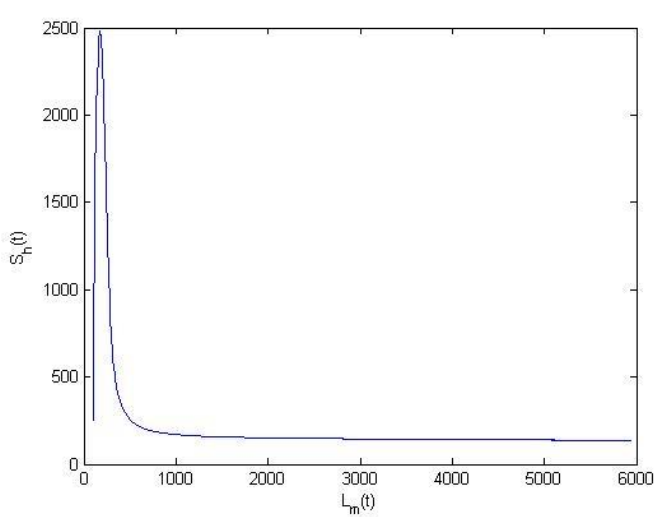

(j)

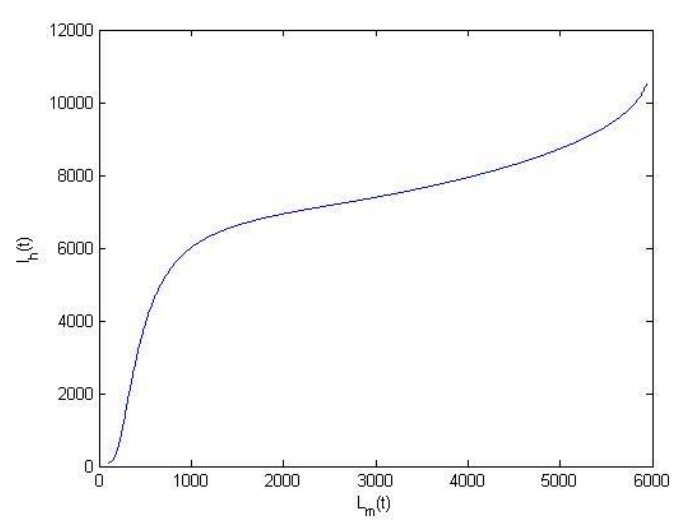

(1)

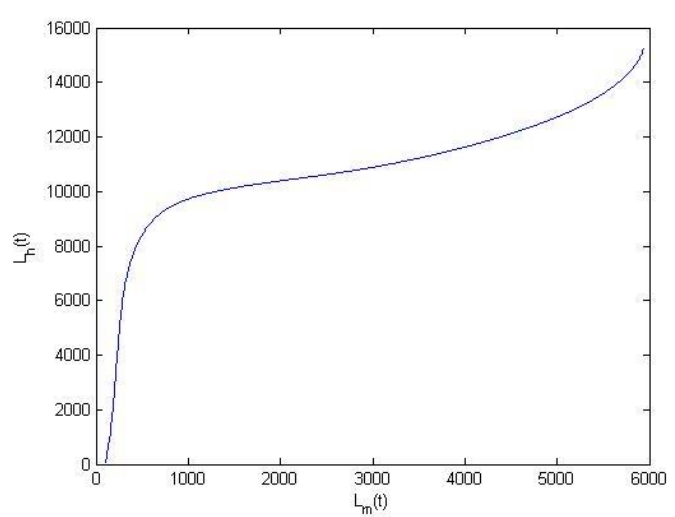

(k)

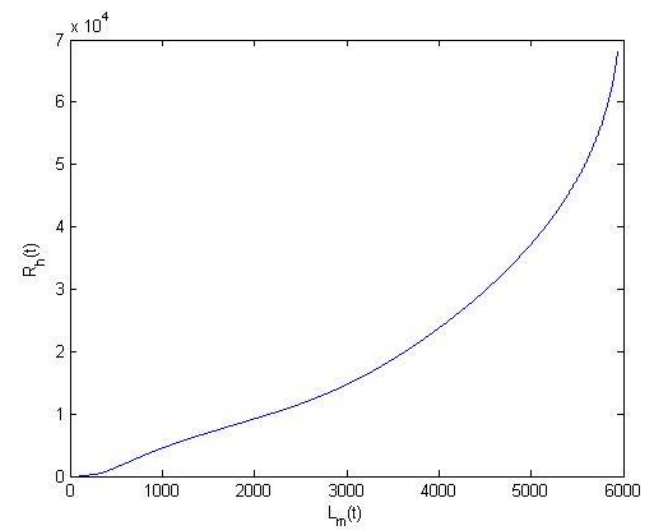

$(\mathrm{m})$

Fig. 2 Graphical behavior of (h) $I_{m}(t)$, (i) $R_{m}(t)$, (j) $S_{h}(t)$, (k) $L_{h}(t)$, (l) $I_{h}(t)$ and (m) $R_{h}(t)$ with $L_{m}(t)$ for $\alpha=0.85$ and for $0<t<6000$.

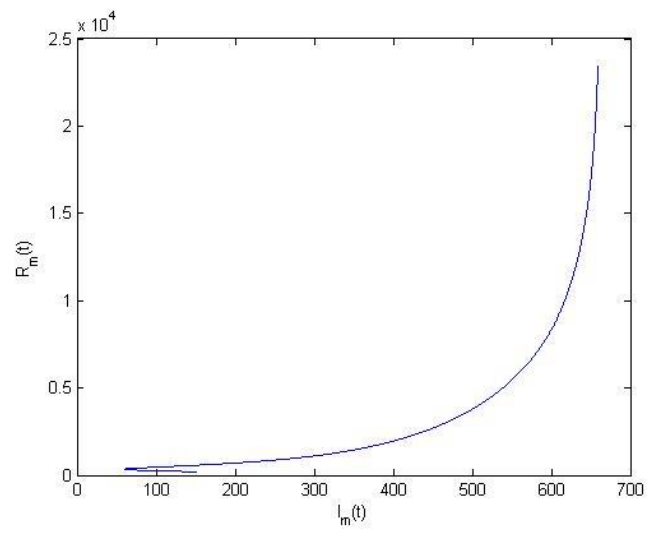

(n)

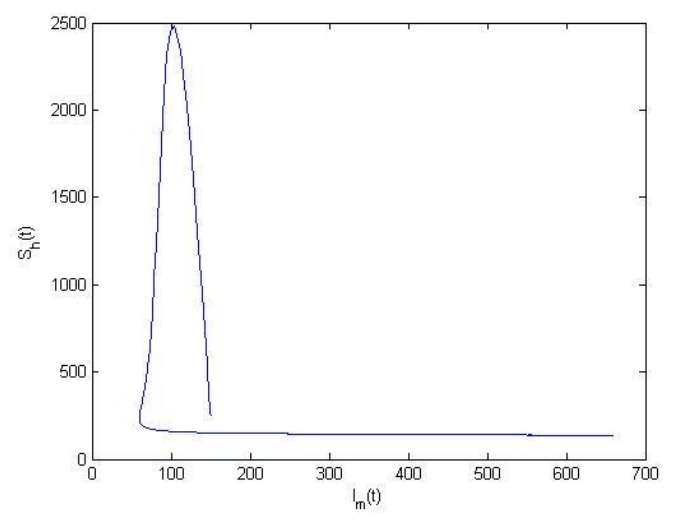

(o) 


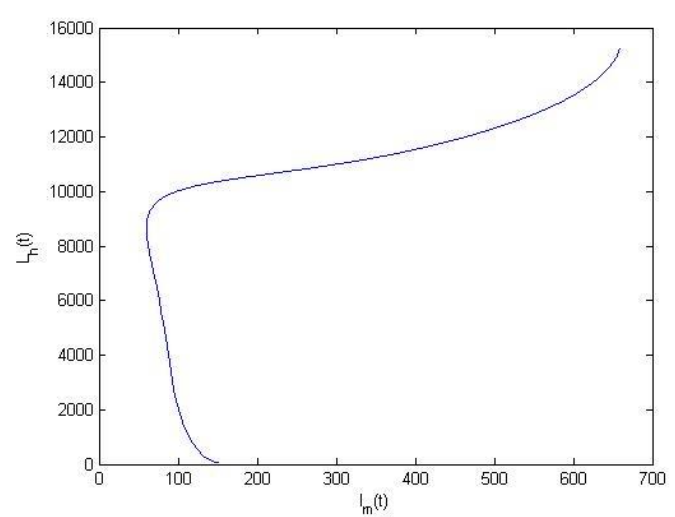

(p)

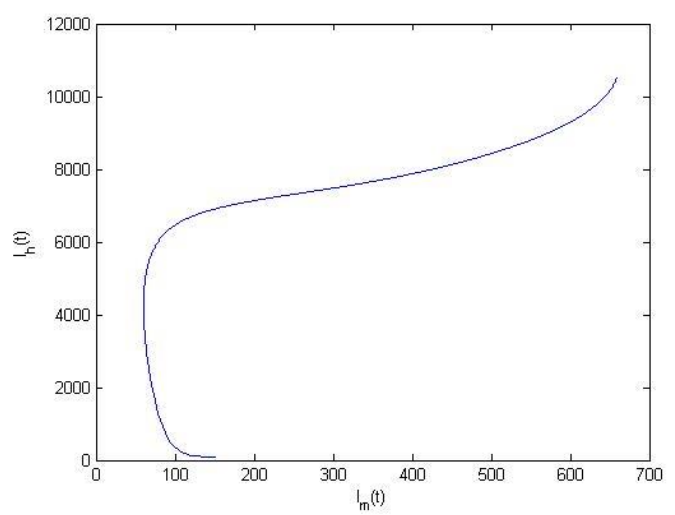

(q)

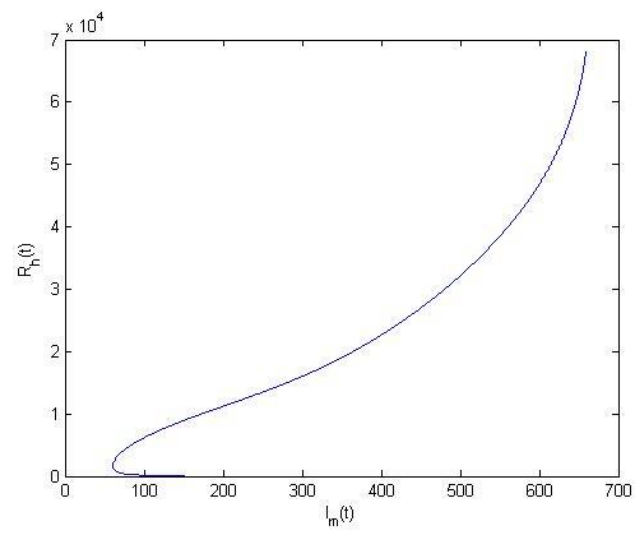

$(\mathrm{r})$

Fig. 3 Graphical behavior of (n) $R_{m}(t)$, (o) $S_{h}(t)$, (p) $L_{h}(t)$, (q) $I_{h}(t)$ and (r) $R_{h}(t)$ with $I_{m}(t)$ for $\alpha=$ 0.85 and for $0<t<700$.

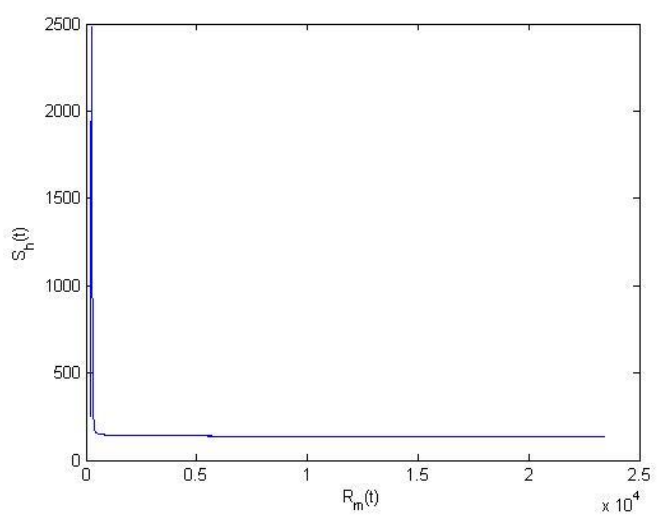

(s)

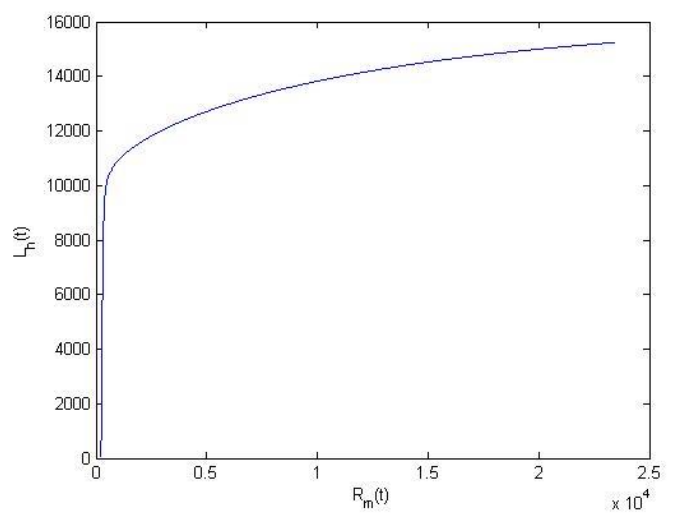

$(\mathrm{x})$ 


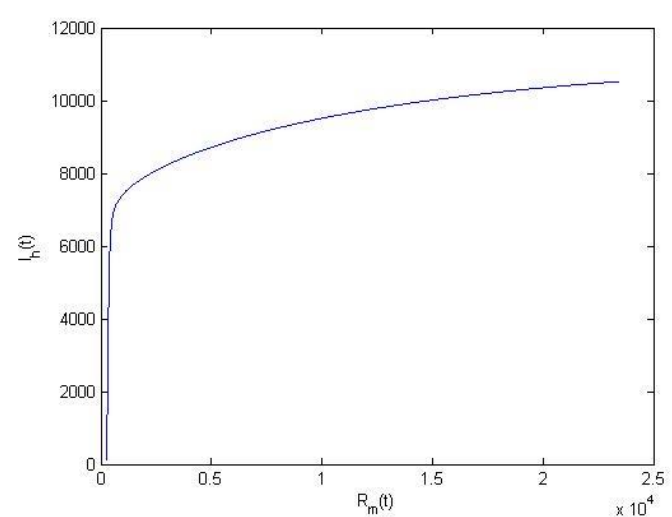

(y)

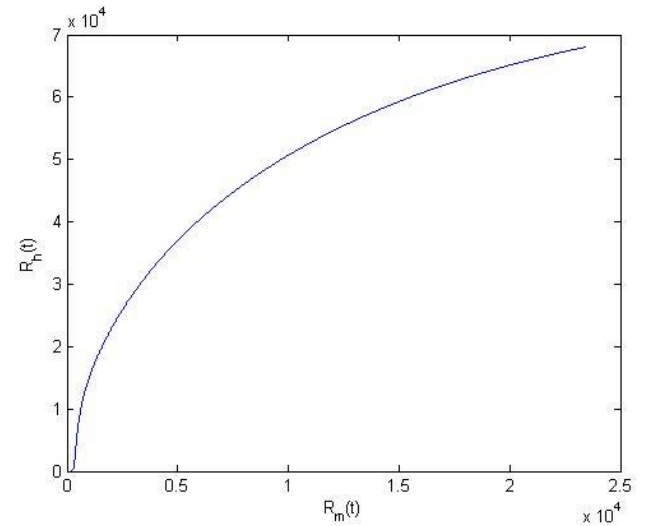

(z)

Fig. 4 Graphical behavior of (s) $S_{h}(t),(\mathrm{x}) L_{h}(t)$, (y) $I_{h}(t)$ and (z) $R_{h}(t)$ with $R_{m}(t)$ for $\alpha=0.85$ and for $0<t<2.5 \times 10^{4}$.

\section{Conclusion}

In the current work, we stretched out the Coronavirus model to fragmentary requests utilizing the CaputoFabrizio partial subordinate. The model equilibria and central expansion number are examined. The presence and uniqueness of the response for the Coronavirus model of the care model with CF subordinate are exhibited in detail. Some mathematical propagations are finished to explore the effect of incomplete solicitations. From mathematical amusements, one can see that when the fragmentary solicitation of subordinate $\alpha$ ([22-28]) reduces, the CF auxiliary gives even more naturally possible direct about the dynamic of pine wither contamination. Thusly, we contemplated that the as of late incomplete auxiliary is important for showing such wonders. Also, from the graphical social we surmise that the proposed halfway solicitation model gives more extreme and progressively versatile results when differentiated and the contrasting entire number solicitation money related models of care model.

\section{Availability of data and material}

No data were used in this study.

\section{Competing interests}

The authors have no interests competing.

\section{Funding}

No funding

\section{Authors' contributions}

All authors designed the study, developed the methodology, performed the analysis, and wrote the manuscript. All authors read and approved the final manuscript.

\section{Acknowledgements}

The authors are thankful to editors and referees for their valuable help.

\section{*Authors' information (optional)}

${ }^{1}$ Department of Mathematics, Faculty of Science, Taif University, Saudi Arabia

${ }^{2}$ Department of Mathematics, Faculty of Science, Zagazig University, Zagazig, Egypt

${ }^{3}$ El-Obour Higher Institute of Engineering and Technology, El-Obour, Qalyubia, Egypt

${ }^{4}$ Departement of Mathematics and Engineering Physics, Faculty of Engineering, Mansoura University,

Mansoura 


\section{References}

1. Helmy, Y. A., Fawzy, M., Elaswad, A., Sobieh, A., Kenney, S. P., \& Shehata, A. A. (2020). The COVID19 pandemic: a comprehensive review of taxonomy, genetics, epidemiology, diagnosis, treatment, and control. Journal of Clinical Medicine, 9(4), 1225.

2. Sanche, S., Lin, Y. T., Xu, C., Romero-Severson, E., Hengartner, N., \& Ke, R. (2020). Early ReleaseHigh Contagiousness and Rapid Spread of Severe Acute Respiratory Syndrome Coronavirus 2.

3. World Health Organization. (2020). Coronavirus disease 2019 (COVID-19) situation reports. 2020.

4. Luo, W., Majumder, M., Liu, D., Poirier, C., Mandl, K., Lipsitch, M., \& Santillana, M. (2020). The role of absolute humidity on transmission rates of the COVID-19 outbreak.

5. Li, Q., Guan, X., Wu, P., Wang, X., Zhou, L., Tong, Y \& Xing, X. (2020). Early transmission dynamics in Wuhan, China, of novel coronavirus-infected pneumonia. New England Journal of Medicine.

6. Zhou, P., Yang, X. L., Wang, X. G., Hu, B., Zhang, L., Zhang, W. \& Chen, H. D. (2020). Discovery of a novel coronavirus associated with the recent pneumonia outbreak in humans and its potential bat origin. BioRxiv.

7. Gralinski, L. E., \& Menachery, V. D. (2020). Return of the Coronavirus: 2019-nCoV. Viruses, 12(2), 135.

8. Garcia, M., Lipskiy, N., Tyson, J., Watkins, R., Esser, E. S., \& Kinley, T. (2020). Centers for Disease Control and Prevention 2019 novel coronavirus disease (COVID-19) information management: addressing national health-care and public health needs for standardized data definitions and codified vocabulary for data exchange. Journal of the American Medical Informatics Association, 27(9), 14761487.

9. Munster, V. J., Koopmans, M., van Doremalen, N., van Riel, D., \& de Wit, E. (2020). A novel coronavirus emerging in China-key questions for impact assessment. New England Journal of Medicine, 382(8), 692-694.

10. Chen, S., Yang, J., Yang, W., Wang, C., \& Bärnighausen, T. (2020). COVID-19 control in China during mass population movements at New Year. The Lancet, 395(10226), 764-766.

11. Ivorra, B., \& Ramos, A. M. (2020). Application of the Be-CoDiS mathematical model to forecast the international spread of the 2019-20 Wuhan coronavirus outbreak. ResearchGate Preprint, 9, 1-13.

12. Yang, C., \& Wang, J. (2020). A mathematical model for the novel coronavirus epidemic in Wuhan, China. Mathematical Biosciences and Engineering, 17(3), 2708-2724.

13. Caputo, M., \& Fabrizio, M. (2015). A new definition of fractional derivative without singular kernel. Progr. Fract. Differ. Appl, 1(2), 1-13.

14. Khan, M. A., Ullah, S., Okosun, K. O., \& Shah, K. (2018). A fractional-order pine wilt disease model with Caputo-Fabrizio derivative. Advances in Difference Equations, 2018(1), 410.

15. Ullah, S., Khan, M. A., \& Farooq, M. (2018). A new fractional model for the dynamics of the hepatitis B virus using the Caputo-Fabrizio derivative. The European Physical Journal Plus, 133(6), 237.

16. Dokuyucu, M. A., Celik, E., Bulut, H., \& Baskonus, H. M. (2018). Cancer treatment model with the Caputo-Fabrizio fractional derivative. The European Physical Journal Plus, 133(3), 92.

17. Singh, J., Kumar, D., \& Baleanu, D. (2018). On the analysis of fractional diabetes model with exponential law. Advances in Difference Equations, 2018(1), 1-15.

18. Khan, M. A., \& Atangana, A. (2020). Modeling the dynamics of novel coronavirus (2019-nCov) with fractional derivative. Alexandria Engineering Journal.

19. Chen, T. M., Rui, J., Wang, Q. P., Zhao, Z. Y., Cui, J. A., \& Yin, L. (2020). A mathematical model for simulating the phase-based transmissibility of a novel coronavirus. Infectious diseases of poverty, $9(1)$, $1-8$.

20. Bonyah, E., Sagoe, A. K., Kumar, D., \& Deniz, S. (2020). Fractional Optimal Control Dynamics of Coronavirus Model with Mittag-Leffler Law. Ecological Complexity, 100880. 
21. Losada, J., \& Nieto, J. J. (2015). Properties of a new fractional derivative without singular kernel. Progr. Fract. Differ. Appl, 1(2), 87-92.

22. Mahdy, A. M. S., \& Youssef, E. S. M. (2020). Numerical solution technique for solving isoperimetric variational problems. International Journal of Modern Physics C, 2150002.

23. Khader, M. M., Sweilam, N. H., Mahdy, A. M. S., \& Moniem, N. A. (2014). Numerical simulation for the fractional SIRC model and influenza A. Applied Mathematics \& Information Sciences Appl. Math. Inf. Sci, 3, 1-8.

24. Othman, M. I., Mahdy, A. M. S., \& Farouk, R. M. (2010). Numerical solution of 12th order boundary value problems by using homotopy perturbation method. Journal of Mathematics and Computer Science, 1(1), 14-27.

25. Mahdy, A. M. S. (2021). Numerical solutions for solving model time-fractional Fokker-Planck equation, Numerical Methods for Partial Differential Equations, 36(2), 1120-1135.

26. Amer, Y. A., Mahdy, A. M. S., \& Youssef, E. (2018). Solving systems of fractional nonlinear equations of Emden Fowler type by using sumudu transform method. Glob. J. Pure Appl. Math, 14(1), 91-113.

27. Amer, Y. A., Mahdy, A. M. S., Shwayaa, R.T., \& Youssef, E. (2018). Laplace transform method for solving nonlinear biochemical reaction model and nonlinear Emden-Fowler System, Journal of Engineering and Applied Sciences, 13(17), 7388-7394.

28. Mahdy, A. M. S., Amer, Y. A., MS Mohamed \& Youssef, E. (2020). General fractional financial models of awareness with Caputo-Fabrizio derivative, Advances in Mechanical Engineering. 12(11) 1-9.

29. Abdel-Halim Hassan, I.H., Othman, M.I.A., Mahdy, A.M.S. (2009). Variational iteration method for solving: Twelve order boundary value problems. International Journal of Mathematical Analysis, 3(1316), pp. 719-730.

30. A. M. S. Mahdy, K. Lotfy, M. H. Ahmed, A. El-Bary \& E.A. Ismail, Electromagnetic Hall current effect and fractional heat order for Micro temperature Photo-Excited semiconductor medium with Laser Pulses, Results in Physics, 17, 1-9, (2020).

31. A. K. Khamis, K. Lotfy, A. A. El-Bary, A. M.S. Mahdy, \& M.H. Ahmed, Thermal-piezoelectric problem of a semiconductor medium during photo-thermal excitation, Waves in Random and Complex Media, $1-15,(2020)$.

32. A. M. S. Mahdy, Kh. Lotfy, E. A. Ismail, A. A. Elbary, M. Ahmed \& A.A.A. El-dahdouh, Analytical solutions of time-fractional heat order for a Magneto-Photothermal Semiconductor medium with Thomson effects and initial stress, Results in Physics, 18, 1-11, (2020).

33. A. M. S. Mahdy, Kh. Lotfy, W. Hassan \& A. A. El-Bary, Analytical solution of magneto-photothermal theory during variable thermal conductivity of a semiconductor material due to pulse heat flux and volumetric heat source, Waves in Random and Complex Media, 1-19, (2020).

34. A.M.S. Mahdy, M.S. Mohamed, K.A. Gepreel, A. AL-Amiri,\& M. Higazy, Dynamical characteristics and signal flow graph of nonlinear fractional smoking mathematical model, Chaos, Solitons, and Fractals 141 (2020) 110308.

35. Mahdy, A. M. S., Higazy, M., Gepreel K.A. \& El-dahdouh, A.A.A. (2020) Optimal control and bifurcation diagram for a model nonlinear fractional SIRC, Alexandria Engineering Journal, 59(5), 3481-3501.

36. Gepreel, K. A., Higazy, M. \& Mahdy, A. M. S., (2020). Optimal control, signal flow graph, and system electronic circuit realization for nonlinear Anopheles Mosquito model, International Journal of Modern Physics C (IJMPC), 31(9), (2020), 1-18.

37. Diethelm K. and Freed A.D. The FracPECE subroutine for the numerical solution of differential equations of fractional order, (1999). p. 57-71.

38. Garrappa R. On linear stability of predictor-corrector algorithms for fractional differential equations. Inter. J. Comput. Math., (2010), 87(10), 2281-90. 
39. Chiou SDJC and Lin YC. Modified Adams-Moulton predictor-corrector method in solving multibody dynamical systems, Mech. Struct. Mach., (2000) 28, 201-208.

40. K. A. Gepreel, M. S. Mohamed, H. Alotaibi and A. M. S. Mahdy. Dynamical behaviors of nonlinear Coronavirus (COVID-19) model with numerical studies, CMC: Computers, Materials \& Continua, (2021), 67(1), 675-686.

41. A. M. S. Mahdy, K. A. Gepreel, L. Khaled and A. A. El-Bary, A numerical method for solving the Rubella ailment disease model, International Journal of Modern Physics C (IJMPC), (2021), 1-18. 
Figures

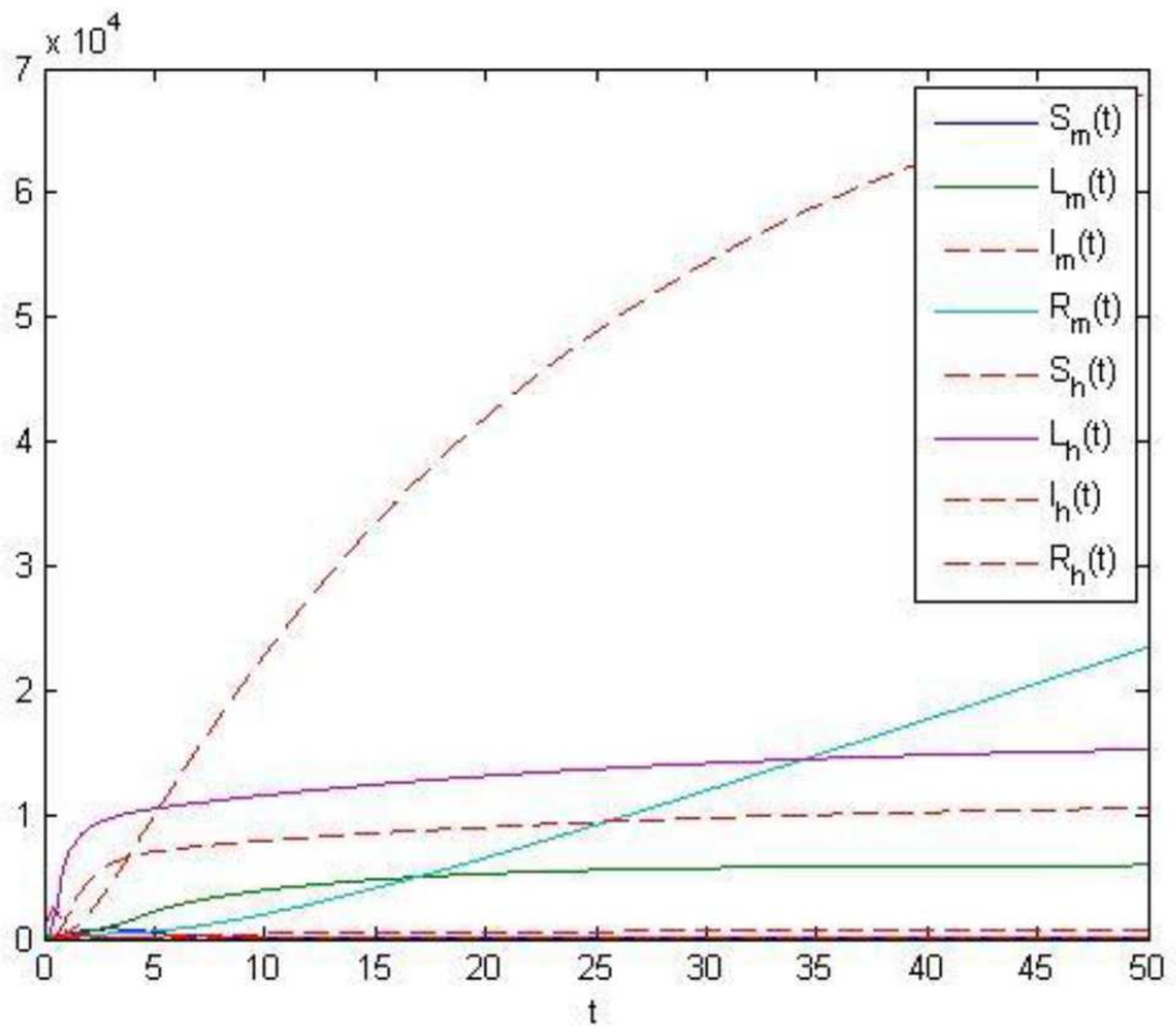

Figure 1

Different value of the parameters for $\bigotimes=0.85$ and for $0<\rrbracket<50$. 

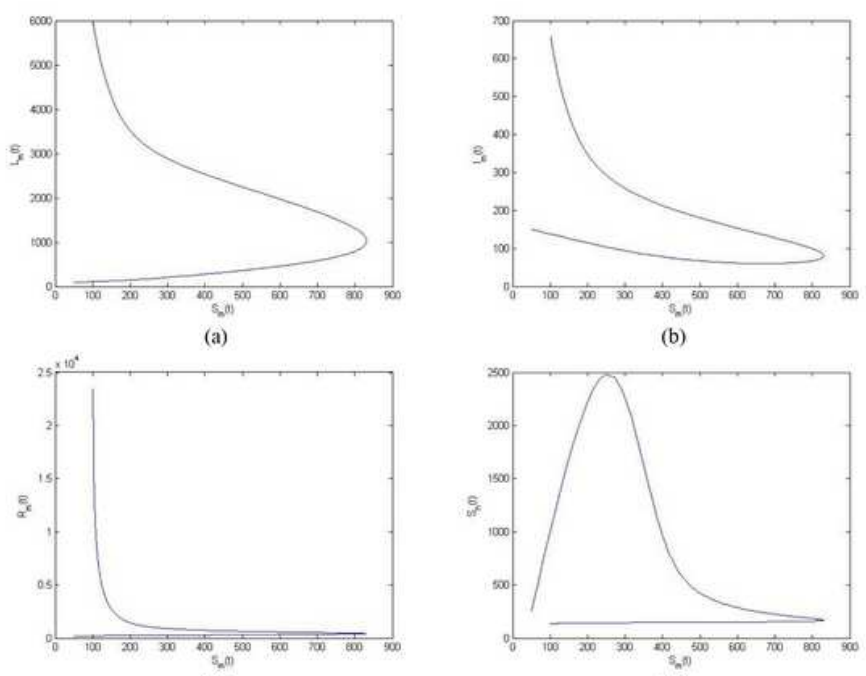

(c)

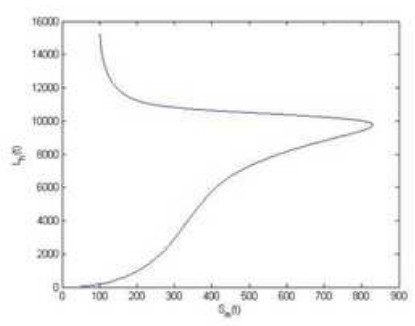

(e)

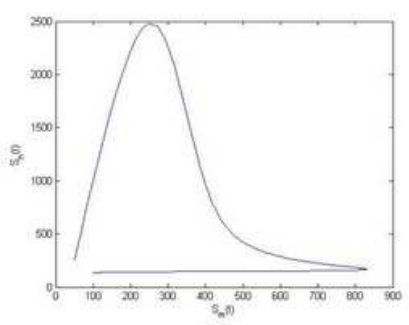

(d)

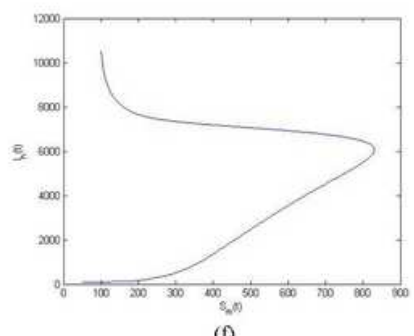

(f)

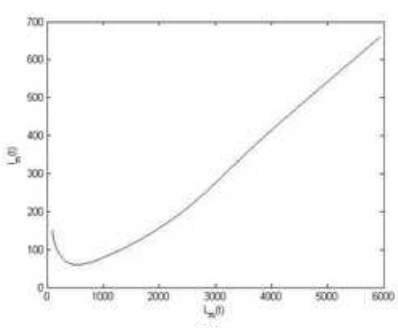

(h)

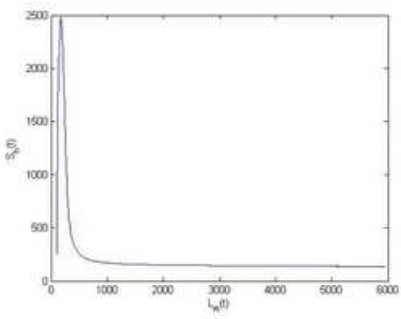

(j)

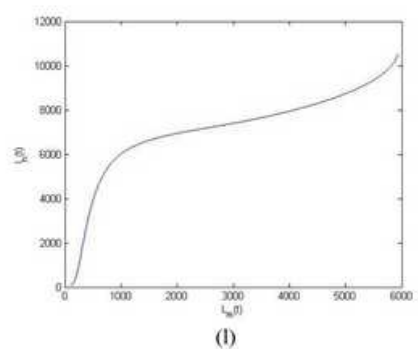

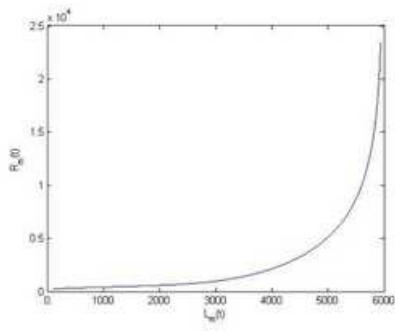

(i)

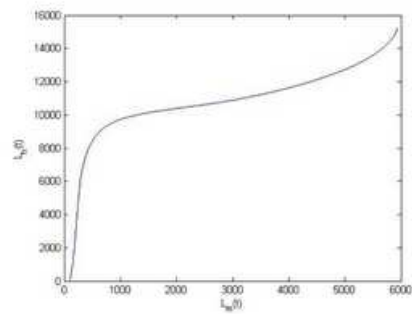

(k)

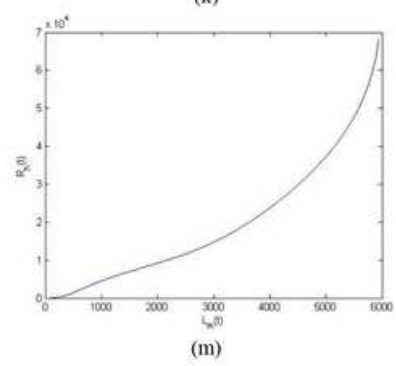

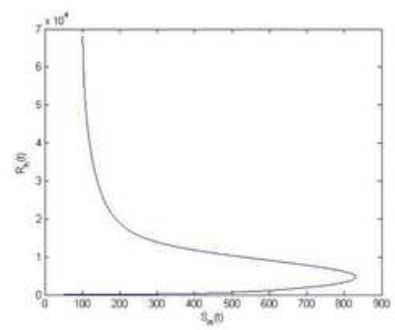

(g)

Figure 2

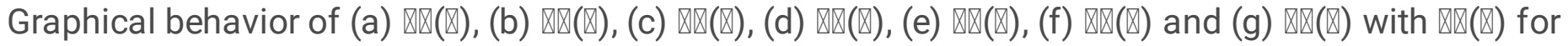

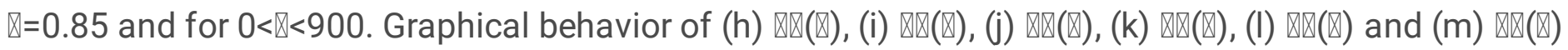

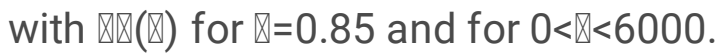




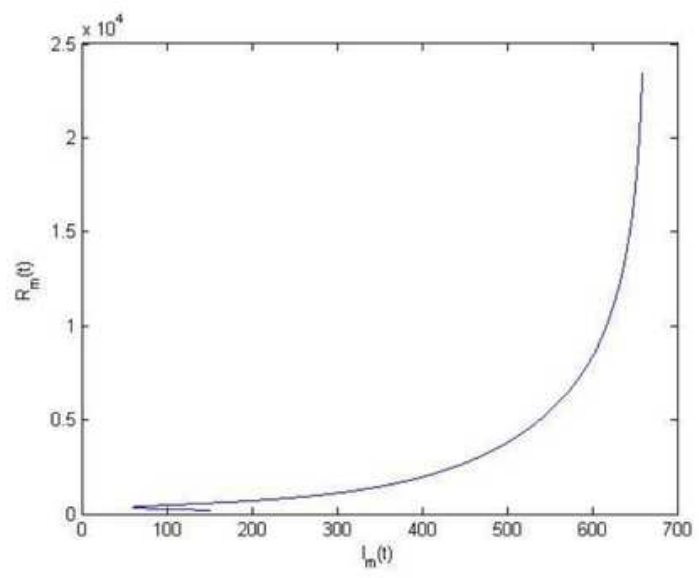

(n)

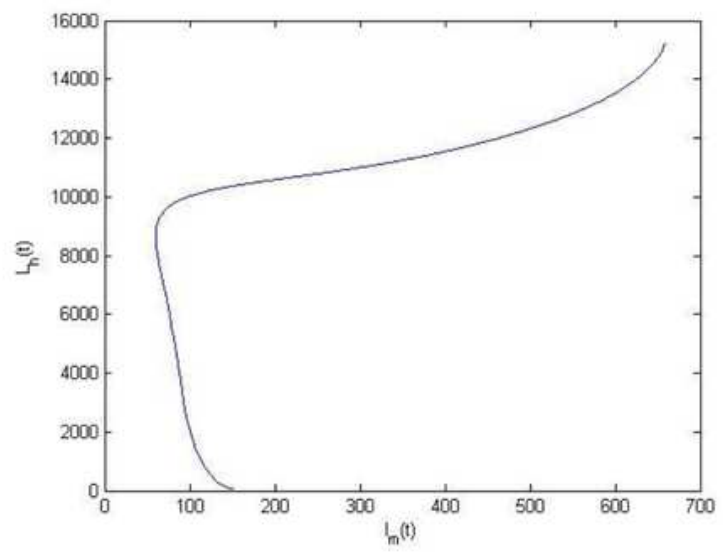

(p)

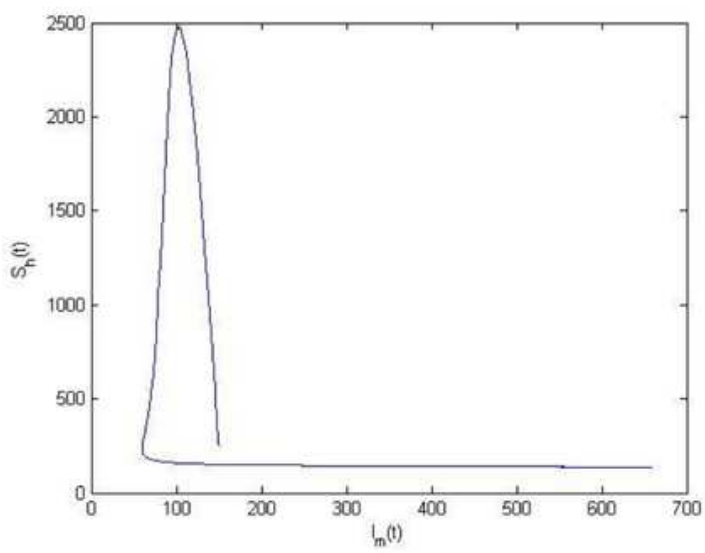

(0)

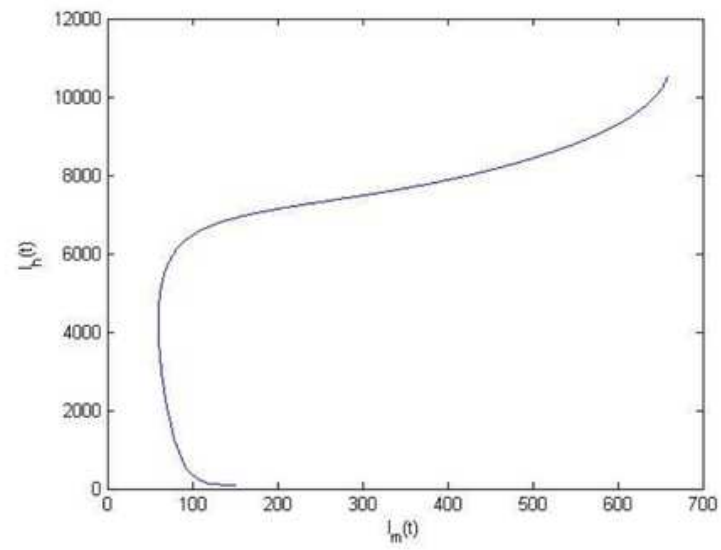

(q)

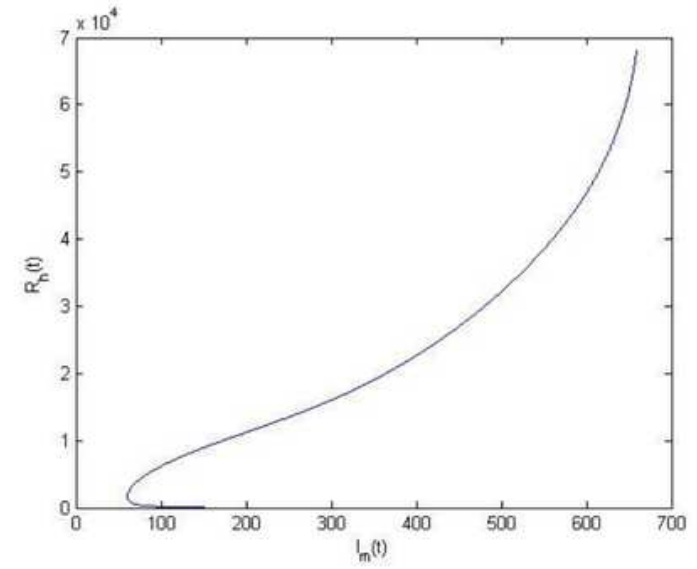

(r)

Figure 3

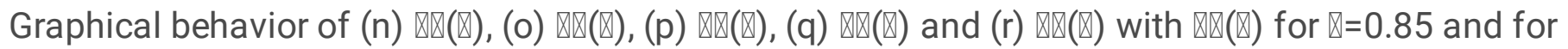

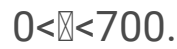




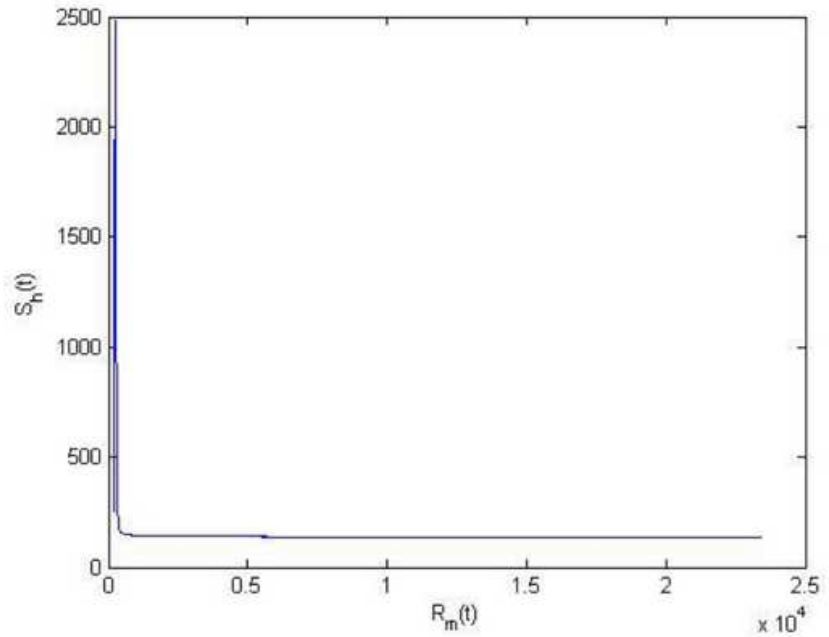

(s)

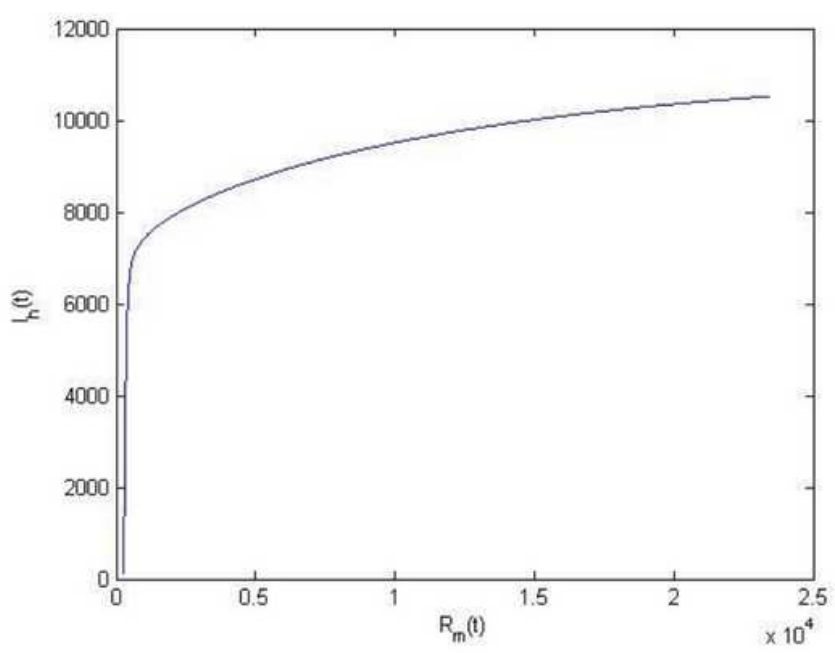

(y)

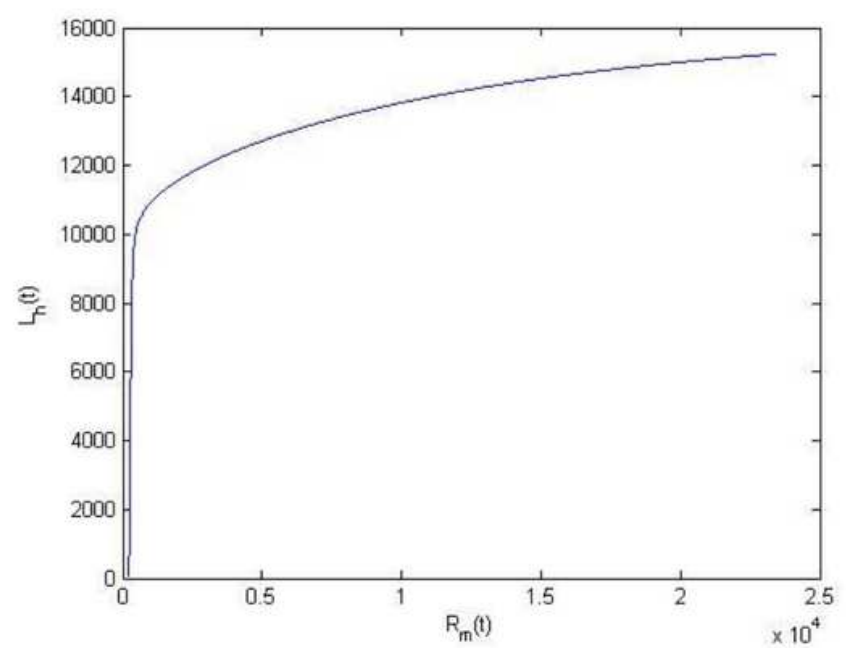

(x)

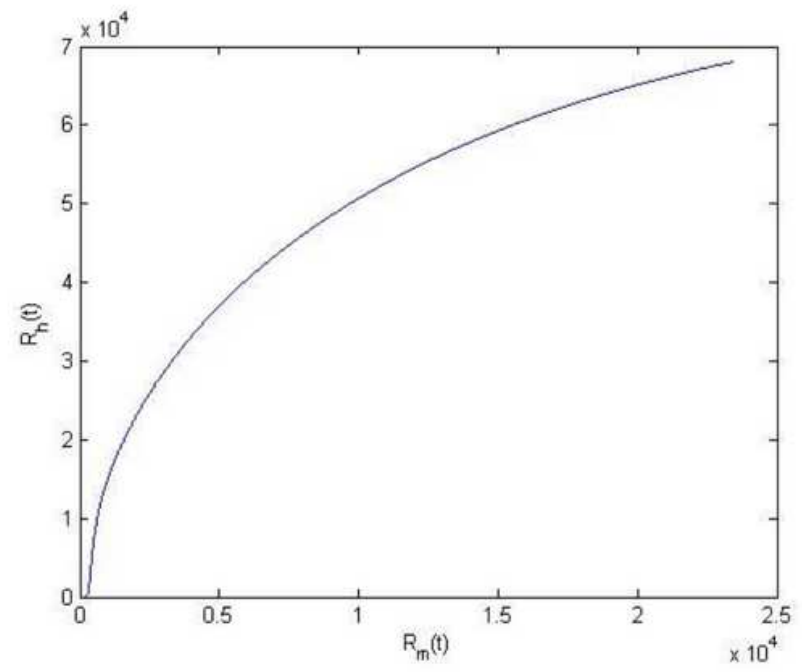

(z)

\section{Figure 4}

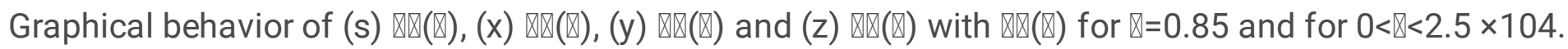

